\title{
On Buoyancy Driven Mixing by Volumetric Microwave Energy Deposition
}

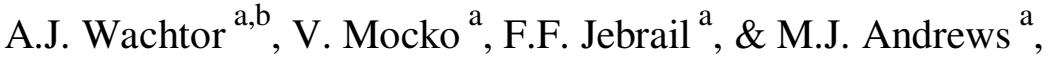 \\ ${ }^{\mathrm{a}}$ Los Alamos National Laboratory, Los Alamos, NM 87545 \\ ${ }^{\mathrm{b}}$ Point of contact: Adam J. Wachtor, ajw@lanl.gov, 505-665-5364
}

\begin{abstract}
Investigation of buoyancy driven mixing by volumetric energy deposition is of particular interest to inertial confinement fusion research. This contribution describes a new microwave facility and an experiment to study buoyancy driven mixing of miscible fluids by volumetric energy deposition. A light weakly-polar fluid initially rested on top of a heavier and higher polarity fluid. As the fluid system was subjected to microwave radiation, less microwave energy was deposited into the weakly-polar fluid than the higher polarity fluid; thus, the bottom fluid was preferentially heated, and its density decreased due to thermal expansion. With continued microwave heating, the density of the bottom fluid dropped below the density of the upper fluid, creating a Rayleigh-Taylor unstable configuration, and, subsequently, buoyancy driven mixing. The miscible pair of toluene and tetrahydrofuran was chosen for the volumetric energy deposition experiments presented. Initially, single fluid microwave heating experiments, for which the source term in the heat equation was varied by variations in the fluid volume, were performed to provide calibration of a mathematical model. The model predicted the neutral stability point of the system, which facilitated experimental design and understanding. Measurements of the mixing layer width from this two-fluid mixing experiment are compared with results from a self-similar analysis of the governing equations.
\end{abstract}

Keywords: microwaves; mixing; Rayleigh-Taylor; unstable stratification; volumetric energy deposition 


\section{Introduction}

Buoyancy driven mixing, such as that resulting from Rayleigh-Taylor (RT) instability [1, 2, 3], has been a focus of research because of its importance to challenging engineering problems such as inertial confinement fusion (ICF), and also because of its relevance to many environmental and technology applications (e.g. geological storage of $\mathrm{CO}_{2}$ [4], droplet breakup $[5,6])$. The application of interest for this work is ICF, in which fusion reactions are achieved by heating and compressing a fuel target, typically in the form of a capsule filled with deuterium and tritium. One of the barriers known to hinder fusion ignition in ICF is buoyancy driven mixing between material layers which occurs during the compression phase of fuel capsule heating [7]. Although the temperatures in ICF experiments are great enough to turn the capsule and its contents into plasma, the phenomenon of buoyancy driven mixing can be profitably studied with fluids governed by the Navier-Stokes equations to gain insight into material mixing which may still contribute to improved ICF designs. Understanding of RT instabilities, and the resulting mixing process, has grown through experimental [8], computational [9-11], and theoretical [12] studies. However, such studies do not address the effects of continuous heating and time dependent density differences which are present in ICF experiments.

Joshi et al. [13] recognized that RT mixing can be driven by properly forcing a dielectric fluid with an electric field. Recently, Wachtor et al. [14] demonstrated that such mixing is possible through the deposition of microwave energy in two miscible dielectric fluids. The physics of how the development of buoyancy driven mixing is affected by volumetric energy deposition (VED) is poorly understood, and has only recently started to be explored [14]. It is believed that VED can aid material mixing processes through buoyancy (pressure gradient) acting on the variation in density. Furthermore, VED may enhance mixing rates (stirring and molecular), and can thus significantly affect local reaction rates and efficiency in chemically reactive systems [15-17]. As such, there is a need for experimental data to validate mathematical models of the VED process.

A microwave experimental facility is particularly well suited for VED applications. Unlike other heating methods, such as convective heating, microwave heating occurs throughout the entire volume of dielectric materials, resulting in a high heating uniformity, and more precise temperature regulation. In this microwave VED study, the substance stored internal energy 
while undergoing a given temperature change, but without undergoing a phase change, i.e. the RT instability was generated below the boiling points of the fluids used. The interaction of two dielectric fluids under microwave heating was examined by Kennedy et al. [16, 17], however, only immiscible fluids were examined. The work of [14] was the first investigation of mixing of two miscible fluids by VED, and set the stage for the current study. The present experiment was initially setup in a RT stable configuration with a lighter, weakly-polar fluid at rest above a heavier, higher polarity (more microwave absorbing) fluid. Preferential microwave heating of the bottom fluid layer caused the bottom density to drop quicker than that of the top fluid. The thermal expansion of the bottom fluid layer caused the system to transition to a RT unstable configuration, and initiated buoyancy driven mixing, as depicted in Figure 1.

In general, the power balance of an ICF capsule involves the energy volumetrically deposited by the fusion products (alpha particles and neutrons), and losses due to mechanical work, radiation, and thermal conduction. The current VED experiment has analogous physical processes to ICF experiments, in that microwave radiation volumetrically deposits energy into the system, and gravity provides a pressure gradient akin to the pressure gradient generated in ICF experiments as the capsule ablates. Thus, the VED experiment can serve as a model that captures two of the principle physical effects that dominate the ICF fusion process.

This work is an advancement of the successful proof-of-concept presented in [14]. Improvements to the design of the experimental facility and procedure are presented in $\S 2$, along with information about the fluids used for the experiment and data acquisition. Single fluid calibration experiments are presented in $\S 3$ along with the mathematical model used to determine fluid temperature variation under microwave heating, and to predict the neutral stability point of the system - careful consideration is given to the limits of the microwave absorption length being much shorter than the vessel, or much longer. Results from the twofluid mixing experiments are given in $\S 4$, followed by conclusions.

\section{Experimental Facility}

\subsection{Microwave Instrument}


The proof-of-concept experiment, detailed in [14], was performed in a CEM Discover benchtop microwave, which was found to have several deficiencies for VED mixing experiments. The most significant drawback of the Discover microwave was that it was designed for efficient chemical synthesis, and therefore had a non-uniform microwave field; fluid heating rates were found to be sensitive to the height within the microwave cavity and total load contained in the cavity. The cavity of the CEM Discover was also small, $\sim 500 \mathrm{~cm}^{3}$, limiting the total volume of fluids used in the experiment. Moreover, optical access into the Discover was limited, with a maximum circular view of the mixing vessel corresponding to $\sim 1.0$ $\mathrm{cm}$ diameter.

With the above shortcomings in mind, the present experimental setup used a BP-210 large format microwave from Microwave Research and Applications, Inc. The BP-210 has a maximum output power of 2,100 Watts and an operating frequency of $2.45 \mathrm{GHz}$. Its rectangular cavity has dimensions of $53.5 \mathrm{~cm}$ (width) x $33.0 \mathrm{~cm}$ (length) x $25.1 \mathrm{~cm}$ (height). Use of this microwave permitted experiments to be performed with larger fluid volumes, and measurements were assisted by multiple access ports. The experimental setup can be seen in Figure 2. Probe access ports on the top of the microwave were used to insert two fiber optic thermometers into a fluid containment vessel, both described below, to collect temperature data. The microwave was modified to include three large optical access ports, one on each side and another on the door, with $3.8 \mathrm{~cm}$ diameter. The experiments reported below used the two side ports to image the mixing process. - see $\S 2.4$ for more details on image collection.

The power absorbed by a fluid, $P$, during a set time, $\Delta t$, is calculated as:

$$
P=M C_{p} \Delta T / \Delta t
$$

where $\Delta T$ is the difference measured in the temperature, $T$, over $\Delta t$ for a known mass of fluid, $M$, with specific heat capacity $C_{p}$. Water was used as the fluid for calibration of the microwave power, per the manufacturer's recommended procedure. Calibration measurements were performed using one $\mathrm{kg}$ of water and a heating time of one minute. Each measurement was repeated four times for microwave dial positions 40-60, Figure 3. Manufacturer specifications indicated that the actual power is within $+/-50 \mathrm{~W}$ of the calibrated power. However, the standard deviation for each dial position was found to be within $10 \%$ of the mean value for that position, Figure 3, resulting in power deviations from the mean that were smaller than the 
manufacturers guidelines for calibrated powers less than $500 \mathrm{~W}$, i.e. dial positions 50 or less. All measurements presented below were taken at dial position 45, corresponding to an average calibrated power of $P_{\text {cal }}=333 \mathrm{~W}$ with standard deviation of $28.5 \mathrm{~W}$.

\subsection{Fluid vessel and filling system}

A quartz vessel of inner dimensions $4.4 \mathrm{~cm}$ (width) x $4.6 \mathrm{~cm}$ (length) x $4.6 \mathrm{~cm}$ (height) and wall thickness of $\sim 0.2 \mathrm{~cm}$ was used as the fluid container for all measurements. Quartz was chosen for its optical transparency, as well as its high microwave transparency. This vessel was designed so that the rectangular section transitioned to a $7.0 \mathrm{~cm}$ diameter cylindrical lip at the top to minimize spillage when removing the vessel from the microwave cavity, see Figure 2.

The vessel also had a quartz filling tube permanently attached in a bottom corner of the vessel with a diameter of $0.8 \mathrm{~cm}$. To run a single fluid experiment, the vessel was simply filled with the desired volume of the fluid. However, to run a two-fluid experiment, the vessel was first filled with the desired volume of initially light fluid, and then set into the microwave atop low microwave absorbing aluminosilicate boards that served to bring the vessel within view of the camera system. Next, a silicone fill hose was connected to a three-way valve used to prevent air bubbles being pumped into the vessel along with the bottom fluid. Care was taken to avoid air bubbles, because bubbles would cause some premixing of the two fluids as they passed through the interface of the two fluids. With the three-way valve attached to the filling tube, the bottom (initially heavy) fluid was pumped in using a NE-4000 syringe pump from New Era Pump Systems, Inc. at a rate of $0.5 \mathrm{~cm}^{3} / \min$ for the first $10 \mathrm{~cm}^{3}$, then increased to $1.0 \mathrm{~cm}^{3} /$ min thereafter to minimize any premixing of the fluids before heating. Once the vessel had been filled with the desired amount of the bottom fluid, the valve was closed and the fill hose removed. A small portion of the bottom fluid, $\sim 1-2 \mathrm{~cm}^{3}$ unavoidably remained within the fill tube during the experiment, but this method of filling was found to be superior to filling the bottom fluid using a long syringe needle inserted into the vessel from above (used in [14]), because premixing of the fluids was reduced.

\subsection{Temperature probes}

Two fiber optic temperature sensors, Omega Engineering Inc. FOBS-2, and two data loggers, Omega Engineering Inc. HHTFO-101, were used to collect temperature data. The sensors have a 
temperature range of $-80{ }^{\circ} \mathrm{C}$ to $250{ }^{\circ} \mathrm{C}$, resolution of $0.1{ }^{\circ} \mathrm{C}$, minimum response time of $0.25 \mathrm{sec}$, and accuracy of $\pm 1{ }^{\circ} \mathrm{C}$. Measured response time of the fiber optic sensors and data loggers was tested by alternatingly placing the probes into the ice bath and boiling water and was reported in [14]. Fiber optic probes were chosen over higher frequency cold-wire probes because fiber optic probes are not affected by the electromagnetic interference of the microwaves, and therefore do not require any signal correction. To determine the consistency between the two probes they were positioned directly next to each other in repeated measurements, resulting in less than $1 \%$ measured temperature difference between the probes.

The probes were positioned at the desired heights within the fluid vessel for temperature collection, and became submerged in the fluids as the vessel was filled. In the two-fluid experiments, this procedure minimized premixing by eliminating the problem of having to disturb the two-fluid system by inserting the probes once the vessel had already been filled, which was found to cause undesirable premixing of the fluids in the experiments of [14].

\subsection{Imaging system}

The side optical access ports were used for imaging the two-fluid system. One port was used to backlight the fluid vessel using a Cole-Parmer low noise illuminator (Model 9741-50). A sandblasted light diffusing glass from Edmunds Optics was inserted into the microwave cavity between the illuminator and the fluid vessel to better even out the backlighting. The two-fluid system was then recorded at 15 frames per second using a NET FOculus IEEE 1394 camera (FO134SB) with $659 \times 494$ pixel resolution. To create the necessary contrast for visualizing the two-fluid mixing process, $0.04 \mathrm{~cm}^{3}$ of food color was added to $50 \mathrm{~cm}^{3}$ of the bottom fluid. Single fluid temperature measurements were collected for the bottom fluid with and without the food dye, and effects of the dye on the heating were found to be negligible (less than $0.5^{\circ} \mathrm{C}$ ).

\subsection{Fluid selection}

A detailed description of the fluids selected for use in the experiment was given in [14], and is only summarized here. Selection of suitable fluids requires that the two fluids have close densities, with the initially greater density, and higher microwave absorbing, fluid on the bottom. The lower density fluid was chosen to be weakly-polar, so that minimal heating would take 
place, and its density would remain nearly constant. From first principles, the power per unit volume absorbed by fluid- $\alpha$ is given by [18-21]:

$$
P_{\alpha} / V_{\alpha}=2 \pi f \varepsilon_{0} \varepsilon_{\alpha}^{\prime \prime} E_{\mathrm{rms}}^{2}
$$

where $V_{\alpha}$ is the total fluid volume, $\varepsilon_{0}$ is the vacuum permittivity, and $f$ and $E_{\text {rms }}$ are the frequency and root mean square value of the electric field strength per unit length within the fluid, respectively, of the microwaves. The dielectric loss, $\varepsilon_{\alpha}^{\prime \prime}$, is the only fluid parameter on the right-hand side of Equation 2, and provides a measure of how much microwave energy will be absorbed by the fluid, and subsequently turned into heat. Thus, the selection of a heavier fluid was restricted to fluids that are miscible with [22, 23], have a greater dielectric loss than, and are close in density to, the lighter fluid.

The variation in density, $\rho$, for the two-fluid system may be viewed in terms of Atwood number, $A t$, defined as:

$$
A t \equiv\left(\rho_{1}-\rho_{2}\right) /\left(\rho_{1}+\rho_{2}\right)
$$

The Atwood number is a dimensionless measure of the density difference between the top and bottom fluids, labelled as 1 and 2, respectively. The stability of the system is indicated by the Atwood number. When a heavy fluid sits below a light fluid, $A t<0$, the system is in a stable configuration as along as the gravitational force is aligned with the density gradient. The system would be unstable if $A t>0$, and is said to be neutrally stable when $A t=0$. By choosing a weakly-polar fluid as the top fluid, the $A t$ of the system was largely governed by changes in temperature/density of the initially heavy, bottom fluid.

The microwave penetration depth, defined as the depth into the fluid where the strength of the field is reduced by $e$ (the base of the natural logarithm), was also considered, and is given by [24]:

$$
L_{P D}=\frac{c}{\omega} \sqrt{\frac{2+2 \sqrt{1+(\tan \delta)^{2}}}{\varepsilon^{\prime}(\tan \delta)^{2}}}
$$

where $\omega$ is the microwave frequency, $c$ the speed of sound, $\tan \delta$ the loss tangent of the fluid, and $\varepsilon^{\prime}$ the dielectric constant of the fluid. The loss tangent is defined in terms of the dielectric 
constant and dielectric loss as $\tan \delta \equiv \varepsilon^{\prime \prime} / \mathcal{E}^{\prime}$, and provides a measure of the electromagnetic energy dissipation within the dielectric fluid. To ensure that heating could be treated as uniform throughout the experimental vessel, the penetration depth was required to be greater than the characteristic length scale of the vessel [25].

The requirements above restrict the number of possible combinations of pure fluids for a successful VED mix experiment. The fluids used here were the same as those in [14], namely toluene (Acros Organics 61046-0010) as the initially light, weakly-polar fluid (fluid 1), and tetrahydrofuran, THF, (Acros Organics 18150-0010) as the initially heavy, higher polarity fluid (fluid 2). This combination of fluids has an initial density difference such that $|A t| \sim 0.01$ and a ratio of the dielectric losses $\varepsilon_{1}^{\prime \prime} / \varepsilon_{2}^{\prime \prime} \sim 0.276$. The penetration depths of toluene and THF are approximately $6.9 \cdot L$ and $3.4 \cdot L$, respectively, where $L=4.5 \mathrm{~cm}$ represents the average characteristic width of the quartz vessel used in the experiment (see $\S 2.2$ ). A summary of the fluid properties is provided in Table 1.

\section{Experiments to Investigate Microwave Heating of a Single Fluid}

\subsection{Experiments}

The time scale for which the microwaves heat the fluid through dipole rotation is much shorter than the time scale for heat diffusion within the fluid. Therefore, the pointwise equation describing single fluid heating can be simplified to:

$$
\frac{d\left(\rho_{\alpha} C_{p_{\alpha}} T_{\alpha}\right)}{d t}=\frac{P_{\alpha}}{V_{\alpha}}=2 \pi f \varepsilon_{0} \varepsilon_{\alpha}^{\prime \prime} E_{\mathrm{rms}}^{2}
$$

where $\alpha$ identifies the fluid being heated. The source term on the right hand side of Equation 5 is equal to the instantaneous power per unit volume absorbed by the fluid. In the work of [14], this source term was varied by increasing the power applied to the fluid system while maintaining a constant volume. This effectively increased the strength of the electric field within the fluid, and therefore increased the power absorbed by the fluid. The work presented here sought to alter the source term by varying the volume and keeping the supplied power fixed - by keeping the dial governing the power supplied to the magnetrons set to Dial Position 45, which 
corresponded to $333 \mathrm{~W}$ from the water calibration in Figure 3. The choice for this particular setting was based on previously successful experiments [14], where two-fluid mixing of the toluene-THF system occurred in less than one minute after microwave power was applied, and the power calibration work described in $\S 2.1$. Fluid volumes were varied in $10 \mathrm{~cm}^{3}$ increments from 30 to $80 \mathrm{~cm}^{3}$, limits set by the vessel and temperature probe setup. All temperatures for single fluid experiments were measured at $1.0 \mathrm{~cm}$ above the vessel bottom, and measurements were repeated 3-8 times. The microwave timer was set to $60 \mathrm{~s}$ and an initial power ramp-up of $\sim 5$ seconds was observed over all experiments, therefore making the total time which power was applied, $t_{f}$, equal to $55 \mathrm{~s}$. The initial temperature, $T_{i}$, of all experiments was that of room temperature $\sim 21^{\circ} \mathrm{C}$.

Deionized water was used as a benchmark for the single fluid heating investigation, the volume variation results for which are shown in Figure 4. It is clear in Figure 5 that the power per unit volume absorbed by the fluid decreased as the volume increased, and subsequently resulted in a lower heating rate. It is worth noting that the power absorbed does not directly correspond to the calibrated power from $\S 2.1$, the average absorbed power determined from Equation 1 across all of the volume variation water experiments was $143 \mathrm{~W}$ with a standard deviation of $15.9 \mathrm{~W}$. The reason for this difference may be attributed to the significant difference in the total dielectric load used in the microwave calibration and the volume variation experiments, and the fact that the BP210 does not have electromagnetic shielding to prevent reflected waves from reaching the magnetrons. Reflected waves - those waves which are reflected off of the cavity walls back to the magnetrons - alter the impedance presented to the magnetrons, resulting in changes to the magnitude and frequency of the electromagnetic field generated by the magnetrons. Thus, the electromagnetic field within the microwave cavity depended on both the power supplied to the magnetrons (determined by the set dial position), and also the total fluid load present in the cavity.

Figure 6 shows the temperature rise of toluene and THF, as the volume of each was varied in $10 \mathrm{~cm}^{3}$ increments from 30 to $80 \mathrm{~cm}^{3}$. It is difficult to distinguish a visible behavior pattern as the volume is increased for either fluid. Indeed, the results for toluene are nearly identical at all volumes. The absorbed power densities for the toluene and THF measurements, Figure 7, were approximately constant for all volumes, indicating that the absorbed power increased with 
increasing volume. This result differs significantly from the case where water was heated, and a clear decrease in absorbed power density was measured. The power dial setting was the same for all measurements, highlighting the important need for differentiation between supplied power, calibrated power, and absorbed power.

The power dial determines the supplied power, but reflected waves that reach the magnetrons can alter their output from the supplied power. Calibrated power is simply a reference power, and does not correspond to the power absorbed by the dielectric load inside the cavity once the load is changed from that used in the calibration. The absorbed power is that which is directly proportional to the rate at which the dielectric is heated, as it gives a measure of the energy lost from the incident electric field to the dielectric, which is subsequently turned into heat.

To understand why the behavior for the absorbed power in the toluene and THF experiments differs from that of water, Equation 5 must be examined further. The right-hand side of Equation 5 describes the average power absorbed by a volume of fluid, $V$, at a given instant. At any instant, the average electric field strength within the fluid volume is then:

$$
E_{\mathrm{rms}}^{2}=\frac{1}{V} \iiint_{V} E^{2}(x, y, z) d V
$$

Two limiting cases for Equation 6 arise depending on dielectric properties of the fluid:

1. Penetration depth is small compared with the characteristic length of the vessel, i.e. all incident energy is absorbed by the fluid.

$$
\frac{P}{V}=2 \pi f \varepsilon_{0} \varepsilon^{\prime \prime} \frac{1}{V} \iiint_{V} E^{2}(x, y, z) d V \approx \frac{\mathrm{C}_{1}}{V}
$$

where $C_{1}$ is equal to a constant that depends on the dielectric properties of the fluid and the frequency and electric field strength of the microwaves.

2. Penetration depth is much greater than the characteristic length of the vessel, i.e. very little energy is deposited to the fluid.

$$
\frac{P}{V}=2 \pi f \varepsilon_{0} \varepsilon^{\prime \prime} \frac{1}{V} \iiint_{V} E^{2}(x, y, z) d V \approx C_{2} E^{2}
$$


where $C_{2}$ is equal to a constant that depends on the dielectric properties of the fluid and the frequency of the microwaves.

Water has a penetration depth smaller than the characteristic length of the vessel, $0.37 \cdot L$, and therefore largely aligns with the first limiting case. However, penetration depths for both toluene and THF are several times greater than the characteristic length of the vessel, and are more closely described by the second limiting case. It was the original desire of this work to demonstrate a change in the energy source term by varying the volume and keeping the absorbed power fixed. However, due to the dielectric properties of toluene and THF and the limiting case pertaining to the average absorbed power density, such variation of the energy source term was not possible while keeping the applied power fixed. Heating rate behavior due to changes in the applied power for these two fluids have already been discussed in [14], and therefore were not pursued further in this work. The variation in volume for toluene and THF presented here therefore represent variations from the average absorbed power density measured across all the experiments for each fluid, see Figure 7.

Measured temperature data was then used to compute the density variation due to heating with a constant applied power for different volumes using the thermal expansion formulas:

$$
\begin{aligned}
& \rho_{1}(t ; V)=0.8864 \mathrm{~g} / \mathrm{cm}^{3}-\left\lfloor 0.0010 \mathrm{~g} / \mathrm{cm}^{3} \cdot{ }^{\mathrm{o}} \mathrm{C}\right\rfloor T_{1}(t ; V) \\
& \rho_{2}(t ; V)=0.9110 \mathrm{~g} / \mathrm{cm}^{3}-\left\lfloor 0.0012 \mathrm{~g} / \mathrm{cm}^{3} \cdot{ }^{\circ} \mathrm{C}\right\rfloor T_{2}(t ; V)
\end{aligned}
$$

These density temperature dependences were measured with a Rudolph Research Analytic DDM 2911 Automatic Density Meter, and ensured an accurate measure of the density variation at the laboratory's $\sim 2150 \mathrm{~m}$ altitude. By converting temperature measurements to density with the above expansion formulas, see Figure 8, Atwood numbers for the two-fluid system were able to be computed, and neutral stability points, $t^{*}$, for the two-fluid system were determined as the time at which $A t=0$. The experimental Atwood numbers, based on the single fluid calibration measurements, and plotted in Figure 9, represent the At which would be measured if the fluids were not allowed to mix. However, THF and toluene were chosen specifically for their miscibility with each other, so therefore this calculation of the $A t$ is only valid up to the neutral stability point where $A t=0$. It may also be valid for densities measured at points within the fluids that are far from the interface for short times following the neutral stability point (i.e. 
locations that have not mixed), but as the fluids begin to mix and thermally equilibrate, the $A t \rightarrow$ 0 again as the system becomes fully molecularly mixed.

The system At behavior is largely determined by how quickly the density of THF decreases, because the density of toluene varies little in these experiments, see Figure 8 . Using the single fluid measurements shown in Figure 8, the neutral stability points for all the volumes measured is predicted to occur within the range of $t^{*} / t_{f} \sim 0.54-0.72$. In particular, the neutral stability point predicted from the At determined from the single fluid measurements for the system containing $40 \mathrm{~cm}^{3}$ of each fluid - the same volumes used in the mixing experiments - occurs at $t^{*} / t_{f} \sim 0.65$. It is informative to visualize this $A t$ behavior in terms of changes to the absorbed power density, Figure 9(b). The results shown in Figure 9(b) reveal that when the absorbed power density increases, the neutral stability point decreases. This is consistent with the behavior demonstrated in [14], which increased the absorbed power density by increasing the applied power.

\subsection{Mathematical modeling}

A simple model describing the temperature rise from the initial temperature, $\Delta T \equiv T-T_{i}$, during single fluid heating was constructed from Equations 1 and 5:

$$
\rho_{\alpha} C_{p_{\alpha}} \frac{d \Delta T_{\alpha}}{d t}=\left(\frac{P_{\alpha}}{V_{\alpha}}\right)_{\text {model }}
$$

where changes to density and specific heat are assumed to be small compared with the changes undergone by the temperature field. The use of $\Delta T$ instead of $T$ allowed for simplification of the initial conditions and final solution form. Only the limiting case pertaining to toluene and THF is addressed in this section, because that is what is directly relevant to the mixing experiment discussed below.

The source terms in Equations 5 and 10 represent the instantaneous power absorbed per unit volume by the fluid. In this work, we consider two forms of the absorbed power density for the model: 
1. Constant absorbed power density, i.e. $(P / V)_{\text {model }}=S$, where $S$ is a constant.

2. Linear absorbed power density, i.e. $(P / V)_{\text {model }}=\tilde{S} t+S$, where $\tilde{S}, S$ are constants.

\subsubsection{Constant absorbed power density}

The constant absorbed power case is considered first. The differential equation and temporal boundary conditions are:

$$
\begin{aligned}
& \rho_{\alpha} C_{p_{\alpha}} \frac{d \Delta T_{\alpha}}{d t}=S_{\alpha} \\
& \Delta T_{\alpha}\left(t=t_{i}\right)=0 \\
& \Delta T_{\alpha}\left(t=t_{f}\right)=\Delta T_{\alpha_{f}} \equiv T_{\alpha_{f}}-T_{\alpha_{i}}
\end{aligned}
$$

The solution to Equation 11 is easily determined to be:

$$
\Delta T_{\alpha} / \Delta T_{\alpha_{f}}=t / t_{f}
$$

for which the source term, $S_{\alpha}$, may have either been determined by simply solving Equation 11 and applying the temporal boundary conditions in Equations 11b,c, or by first determining the power density using Equation 1. This linear solution is plotted with the experimental measurements for THF and toluene in Figure 10. The constant absorbed power model captured the behavior of the toluene temperature profiles well, see Figure 10(b), and had a coefficient of determination of $R_{2}^{2}=0.9948$ averaged over all volume sets. Although the constant absorbed power model also captured the global trends of the measured behavior for THF $\left(R_{2}^{2}=0.9636\right.$ averaged over all volume datasets), Figure 10(a) - dashed line, it is evident that this model omits necessary physics which caused deviations from the model and experimental data.

From the curvature of the measured THF profiles, it is apparent that the absorbed power density for THF changed over time. The reason why the measured absorbed power density changed as THF was heated is likely due to the fact that the dielectric loss is a function of temperature, i.e. $\varepsilon^{\prime \prime}=\varepsilon^{\prime \prime}(T)$. The more the fluid heated up, the greater impact changes to the dielectric loss had on the absorbed power density, not only through its own value, but also by 
potentially altering penetration depth and subsequently the value of $E_{\mathrm{rms}}$ within the fluid as well.

\subsubsection{Linear absorbed power density}

To capture the temperature changes to the absorbed power density observed from the THF measurements that can be attributed to changes in the dielectric loss, the modeled source term was modified to:

$$
\rho_{\alpha} C_{p_{\alpha}} \frac{d \Delta T_{\alpha}}{d t}=\beta \varepsilon_{\alpha}^{\prime \prime}\left(T_{\alpha}\right) \quad \text { where } \quad \beta=\text { constant }
$$

The constant, $\beta$, was estimated using the temporal average of the absorbed power, resulting in the equation set:

$$
\begin{aligned}
& \frac{d \Delta T_{\alpha}}{d t}=\left\langle\frac{\Delta T_{\alpha}}{\Delta t}\right\rangle \frac{\varepsilon_{\alpha}^{\prime \prime}\left(T_{\alpha}\right)}{\left\langle\varepsilon_{\alpha}^{\prime \prime}\right\rangle} \\
& \approx\left\langle\frac{\Delta T_{\alpha}}{\Delta t}\right\rangle \frac{\varepsilon_{\alpha}^{\prime \prime}{ }_{i}}{\left\langle\varepsilon^{\prime \prime}{ }_{\alpha}\right\rangle}\left(1+\frac{1}{\varepsilon_{\alpha_{i}}^{\prime \prime}} \frac{d \varepsilon^{\prime \prime}}{d T} \Delta T_{\alpha}\right) \\
& \Delta T_{\alpha}\left(t=t_{i}\right)=0
\end{aligned}
$$

where $<>$ in the equations above represent a temporal average taken over the entire experimental time. This equation set has the solution:

$$
\begin{aligned}
\Delta T_{\alpha} & =\frac{\varepsilon_{\alpha_{i}^{\prime \prime}}}{d \varepsilon_{\alpha}^{\prime \prime} / d T}\left[\exp \left(\left\langle\frac{\Delta T_{\alpha}}{\Delta t}\right\rangle \frac{1}{\left\langle\varepsilon_{\alpha}^{\prime \prime}\right\rangle} \frac{d \varepsilon^{\prime \prime}}{d T} t\right)-1\right] \\
& \approx \frac{\varepsilon_{\alpha_{i}^{\prime \prime}}}{\left\langle\varepsilon_{\alpha}^{\prime \prime}\right\rangle}\left\langle\frac{\Delta T_{\alpha}}{\Delta t}\right\rangle\left[t+\frac{1}{2}\left\langle\frac{\Delta T_{\alpha}}{\Delta t}\right\rangle \frac{1}{\left\langle\varepsilon_{\alpha}^{\prime \prime}\right\rangle} \frac{d \varepsilon_{\alpha}^{\prime \prime}}{d T} t^{2}\right]
\end{aligned}
$$

Taking the limit of Taylor-series expansion of the solution in Equation 15 as $d \varepsilon_{\alpha}^{\prime \prime} / d T \rightarrow 0$, recovers the solution to the constant absorbed power model, as in that case $\left\langle\varepsilon_{\alpha}^{\prime \prime}\right\rangle \rightarrow \varepsilon_{\alpha_{i}}^{\prime \prime}$ and $\left\langle\Delta T_{\alpha} / \Delta t\right\rangle \rightarrow \Delta T_{\alpha_{f}} / t_{f}$. The exponential solution in Equation 15 is plotted in Figure 10(a) red line - using $\Delta t=2 \mathrm{~s}$, averages determined from the experimental data, and $d \varepsilon_{\alpha}^{\prime \prime} / d T$ 
determined using a linear fit from the data reported in [26, 27]. It is seen that the solution agrees well with the experimental data $\left(R_{2}^{2}=0.9964\right)$, however this solution requires detailed experimental data to compute the averages, as well as knowledge about how the dielectric loss changes with temperature - information which is not always easily found in the literature, nor is the equipment to measure dielectric loss as common to find in a laboratory as methods to measure temperature. Therefore, the Taylor-series expansion of the solution in Equation 15 was used as motivation for modelling the absorbed power density with linear temporal behavior, such as:

$$
\begin{aligned}
& \rho_{\alpha} C_{p_{\alpha}} \frac{d \Delta T_{\alpha}}{d t}=\tilde{S} t+S \\
& \Delta T_{2}\left(t=t_{i}\right)=0 \\
& \Delta T_{2}\left(t=t_{f}\right)=\Delta T_{2_{f}} \equiv T_{2_{f}}-T_{2_{i}} \\
& \Delta T_{2}\left(t=t_{f} / 2\right)=0.58 \Delta T_{2_{f}} \text { from measured data }
\end{aligned}
$$

The solution to Equations 16a-d is found to be:

$$
\Delta T / \Delta T_{f}=1.32 t / t_{f}-0.32\left(t / t_{f}\right)^{2}
$$

This model, Figure 10(a) - blue line, was a great improvement to the constant model, capturing the behavior of the measured data for essentially the entire experiment $\left(R_{2}^{2}=0.9988\right.$ averaged over all volume datasets). Note that this linear model required three temperature measurements over the course of the experiment, i.e. an initial, intermediate, and final temperature, to determine the model constants, whereas the constant model only needed an initial and final temperature value. The intermediate temperature value was arbitrarily chosen at $t / t_{f}=0.5$, so that the model would fit the data better within the intermediate region where the constant model failed. The constant model is actually a special case of this linear model when $\Delta T\left(t=t_{f} / 2\right)=\Delta T_{f} / 2$.

Both of the models require little data for the calibration of the model constants, and therefore can be used to gain insight into the heating behavior when such detailed data, as that presented here, is not available. For example, in a microwave setup which does not have direct probe 
access into the cavity, such that temperature can only be measured at the beginning and end of heating, these models may provide a guide to the temperature behavior in between the measured points.

The neutral stability point determined from the single fluid measurements was $\left(t^{*} / t_{f}\right)_{\text {meas }}=0.64$. The semi-empirical solutions of the model equations, Equations 12 and 17, can be used along with the expansion formulas, Equation 9, to estimate the neutral stability point. Although the global trends of the constant source model and the measurements are similar, the details of the curvature of the temperature profile due to changes in the dielectric properties will not be captured with the constant absorbed power model. Therefore, predictions of the neutral stability point using only the constant absorbed power model, will be greater than the one determined from the single fluid measurements. Using the constant absorbed power model for both toluene and THF gives the predicted value of $\left(t^{*} / t_{f}\right)_{\text {cnst-cnst }}=0.73$, which is approximately $14 \%$ greater than the value determined directly from the temperature measurements. The neutral stability point prediction using the constant model for toluene and linear model for THF was $\left(t^{*} / t_{f}\right)_{\text {cnst-lin }}=0.65$, falling within $2 \%$ of the value predicted from the single fluid measurements.

\section{Two-Fluid Mixing}

Results from the mixing of $40 \mathrm{~cm}^{3}$ of THF with $40 \mathrm{~cm}^{3}$ of toluene at Dial Position 45 ( $330 \mathrm{~W}$ from water calibration) are described in this section. Temperature data collected from three repetitions of the two-fluid mixing experiments are shown in Figure 11, along with data collected for the $40 \mathrm{~cm}^{3}$ single fluid experiments. Temperature probes, in the two-fluid experiment, were positioned vertically at the center of each fluid, e.g. the bottom probe was initially in the middle of THF as shown in Figures 2 and 14a. From Figure 11, it is seen that at early times the temperatures grew at rates similar to those for the single fluids, despite the total dielectric load present in the two-fluid experiments being slightly different than that for the single fluids. The total microwave load in the single fluid THF experiments was $40 \mathrm{~cm}^{3}$ of THF, 
and changed to $40 \mathrm{~cm}^{3}$ of each of the fluids in the two-fluid experiment. However, as the power absorbed by $40 \mathrm{~cm}^{3}$ of toluene was approximately an order of magnitude smaller than that of the same amount of THF, the presence of toluene in the two-fluid experiment had minor effect on the power which THF absorbed. Likewise, the low heating rate of toluene was seen to be independent of the total load, and therefore the presence of THF in the two-fluid experiment only affected the power absorbed by the toluene by a negligible amount.

The sudden departure of the curve for the top probe in the two-fluid experiment from the single fluid toluene curve, is because of mixing, i.e. the mixing layer interface had passed over the probe location. The measured temperature rise for single fluids calibrations of THF and toluene are approximately $26{ }^{\circ} \mathrm{C}$ and $3{ }^{\circ} \mathrm{C}$, respectively. For the two-fluid mixing case the temperature rise measured by both probes initially located in the top and bottom fluid are 18-22 ${ }^{\circ} \mathrm{C}$. The temperature difference between the two probe locations in the mix experiments are $\sim 3$ $4{ }^{\circ} \mathrm{C}$ at the end of the experimental run, see Figure 11a in revised manuscript, and are consistent with the behavior previously seen in [14]. If microwave heating would have been carried out for longer durations, then the temperature difference between the probes would go to zero, just as in [14]. However, the present focus of interest was in the mixing process and not reaching thermal equilibrium for the system. This result provides additional evidence that the fluids are mixing, for if the fluids were immiscible but still able to become RT unstable, then the single fluid calibrations would suggest that the temperature difference between the two probe locations would be $\sim 23{ }^{\circ} \mathrm{C}$. The point for which mixing started was consistently $10 \mathrm{~s}$ before the neutral stability point prediction from the single fluid measurements $\left(0.71 t^{*}\right)$, an aspect that will be discussed below.

Evolution of two-fluid mixing are displayed in Figure 12. Images of the two-fluid mixing process were captured by adding dye to THF, and backlighting the fluid vessel. Consistent with the notion of "bubble-deflation", described by the linear RT stability theory in [14], a long wavelength appeared first and subsequently broke down into successively smaller scales. This feature is caused by the variable $A t$ of the system and transition of the At from negative to positive. A RT linear stability analysis reveals that the most unstable wavelength is proportional to $A t^{-1 / 3}$. Therefore, when $A t<<1$, the largest physical wavelength in the flow will be the most unstable, and as heating continues, the At continues to grow and subsequently smaller modes 
begin to dominate, distinguishing VED generated RT from classical RT $[8,9,14]$. In classical RT, the smallest wavelengths appear first and are the most unstable; they then evolve to larger scale flow features through mode coupling. Analysis of the images is performed after first correcting the images for non-uniform backlighting and shadow effects. Histograms of the initial image, taken before microwave application, and the corrected image are shown in Figure 13. The separated peaks correspond to two initially distinct fluids, as can be seen in the top left of Figure 12. A demonstration of the image correction is presented in Figure 14, where the nonuniformity of the backlighting clearly present in Figure 14b has been corrected in the image seen in Figure 14c.

Although it cannot be easily recognized from the still images in Figure 12, it was witnessed that a convective recirculation pattern forms in the bottom THF layer as it started to heat. The behavior of the THF layer was similar to that of [28], who simulated the flow pattern of a low absorbing fluid as it is being heated in a microwave. This recirculation current rises up through the middle of the layer and falls down the sides. Such motion not only unavoidably causes some mixing to occur at the interface of the two fluids prior to the time at which the system is predicted to become unstable, but also causes a pressure-gradient at the fluid interface. Mixing $10 \mathrm{~s}$ prior to the predicted neutral stability point, as observed above, is attributed to a convolution of these effects. Since THF has a relatively low viscosity, it is believed that selection of a fluid with a higher viscosity will reduce these effects. However, fluid substitution would require that the new fluid still meet all previously stated requirements, and is outside the intended scope of this paper.

The refractive indices of THF and toluene differ by $\sim 0.09$, sufficient to distort light coming from the backlighting source in regions where the light path must intersect heavily convoluted fluid structures. This aspect of the experiment was taken advantage of to obtain a measurement for the temporal evolution of the mixing layer width by isolating those regions, as seen in Figure 14. After the image was thresholded, it was divided into seven vertical subsections, and for each subsection an estimation of the mixing layer width was computed. This was performed by summing over the width of the section to create a curve that identifies the areas of most mix. That curve was then normalized by the peak of the curve (omitting the bottom portion where the mixing layer was not located, but had high peaks because of optical distortions in the THF near 
the bottom of the vessel), and the mixing layer width was then obtained by taking the physical distance between the first and last locations that the normalized curve surpasses $30 \%$ of the peak value. The result of this procedure can be seen in Figure 16.

It is theorized that the VED process will increase the rate of mixing over the traditional $t^{2}$ growth of the mix layer thickness seen in classical RT problems. To examine this, a 0dimensional $k-\varepsilon$ [29] model is considered, which implicitly assumes the gradients of turbulent kinetic energy and dissipation are zero, i.e. diffusion is neglected.

$$
\begin{aligned}
& \frac{\partial k}{\partial t}=\frac{c_{\mu}}{\sigma_{t}} \frac{k^{2}}{\varepsilon} \frac{g}{\rho_{0}} \frac{\left(\rho-\rho_{0}\right)}{W}-\varepsilon \\
& \frac{\partial \varepsilon}{\partial t}=c_{3} \frac{c_{\mu}}{\sigma_{t}} k \frac{g}{\rho_{0}} \frac{\left(\rho-\rho_{0}\right)}{W}-c_{1} \frac{\varepsilon^{2}}{k}
\end{aligned}
$$

where $k$ is the turbulent kinetic energy, $\varepsilon$ is the dissipation, $W$ the mixing layer width, and $c \mu$, $c_{1}$, and $c_{3}$ are coefficients. Temperature measurements in Figure 11 indicate $T \sim t$ prior to the

time at which mixing begins, and if $k \sim t^{4}$ based on self-similarity, then the mixing layer width would have a temporal dependence of $W \sim t^{3}$. Fitting the data for the mixing layer with a $t^{3}$ curve, Figure 16, appears to accurately portray the measured growth of the mixing, demonstrating the growth rate is indeed increased over classical RT growth. It is worth noting that the model equations have been cast as a time varying Atwood number, but could as well be cast as a variable acceleration, and thus this VED experiment can also be thought of a variable acceleration experiment, one in which future work might explore different heating rates as a means to investigate different acceleration histories.

\section{Conclusions}

An overview of a microwave experiment designed to investigate buoyancy driven mixing due to VED, and results from the experiment have been presented. Careful selection of a fluid pair is paramount to a successful microwave based VED experiment. It was found that changes to the fluid load present in the microwave affected the subsequent heating rates greater in fluids with penetration depths smaller than the characteristic vessel length, while those with larger penetration depths resulted in nearly constant power densities. A mathematical model was 
developed to predict the heating rates of single fluid systems, which was used to predict the neutral stability point.

The experiment did have some shortcomings, such as the observed mix time occurring prior to the predicted neutral stability point. Efforts to minimize, or eliminate, the convective recirculation flow pattern in the THF layer that is likely responsible for the neutral stability point offset will be taken in future experiments. However, the present results were consistent with the "bubble deflation" theory of [14], as well as the predicted $W \sim t^{3}$ - providing the first quantitative evidence that the mixing layer growth is increased over the $t^{2}$ classical RT scaling.

Design and testing of the present VED experimental facility has facilitated an investigation of VED mixing. The present large microwave facility has opened the opportunity to increased data collection, i.e. full field imaging resulting in mixing layer width calculations. Future data acquisition will be focused on gathering turbulence measurements of the temperature / density field with fast-response temperature probes and velocity using particle seeding and laser diagnostics.

\section{Acknowledgments}

The authors would like to thank Dr. Marion Vance for his contributions to the imaging setup for the experiment. Los Alamos National Laboratory (LANL) is operated by the Los Alamos National Security, LLC for the U.S. Department of Energy NNSA under Contract No. DE-AC52-06NA25396. This work was made possible by funding from LANL Science Campaign 4.

\section{Nomenclature}

\section{Latin}
At
Atwood number
$S, \tilde{S} \quad$ Heating model constants
$C_{p} \quad$ Specific heat capacity
T $\quad$ Fluid temperature 


$\begin{array}{llll}C_{1}, C_{2} & \text { Constants } & T_{i}, T_{f} & \text { Initial, final fluid temperature } \\ c_{\mu}, c_{1}, c_{3} & \text { Turbulence model coefficients } & t & \text { Time } \\ E & \text { Electric field strength } & t_{i}, t_{f} & \text { Initial, final time of experiment } \\ f & \text { Microwave frequency } & t^{*} & \text { Neutral stability point } \\ g & \text { Acceleration due to gravity } & V & \text { Volume of fluid } \\ k & \text { Turbulent kinetic energy } & W & \text { Mixing layer width } \\ L_{P D} & \text { Penetration depth } & x, y, z & \text { Spatial coordinates } \\ L & \text { Characteristic vessel width } & & \\ P & \text { Absorbed power } & & \end{array}$

\section{Greek}

$\alpha \quad$ Sub index used to identify fluid $\quad \varepsilon^{\prime} \quad$ Dielectric constant

$\delta, \tan (\delta) \quad$ Phase lag angle, loss tangent $\quad \varepsilon^{\prime \prime} \quad$ Dielectric loss

$\varepsilon \quad$ Dissipation of turbulent kinetic energy $\quad \sigma_{t}$ Turbulence model parameter

$\begin{array}{llll}\varepsilon_{0} & \text { Vacuum permittivity } & \rho & \text { Density }\end{array}$

\section{References}

1. Rayleigh, Lord. 1883, Investigation of the character of the equilibrium of an incompressible heavy fluid of variable density, Proc. London Math. Soc., 14, 170-177.

2. Taylor, G.I. 1950, The instability of liquid surfaces when accelerated in a direction perpendicular to their planes, Proc. R. Soc. London A, 201 (1065), 192-196.

3. Sharp, D.H. 1984, An overview of Raleigh-Taylor instability, Phys. D, 12, 3-10. 
4. McGrail, B.P., Schaef, H.T., Ho, A.M., Chien, Y.-J., Dooley, J.J. \& Davidson, C.L. 2006, Potential for carbon dioxide sequestration in flood basalts, J. Geophys. Res., 111 (B12201), $1-13$.

5. Joseph, D.D., Belanger, J. \& Beavers, G.S. 1999, Breakup of a liquid drop suddenly exposed to a high-speed airstream, Int. J. Multiphase Flow, 25 (6), 1263-1303.

6. Varga, C.M., Lasheras, J.C. \& Hopfinger, E.J. 2003, Initial breakup of a small-diameter liquid jet by a high-speed gas stream, J. Fluid Mech., 497, 405-434.

7. Lindl, D. 1995, Development of the indirect-drive approach to inertial confinement fusion and the target physics basis for ignition and gain, Phys. Plasmas, 2 (11), 3933-4024.

8. Andrews, M.J. \& Dalziel, S.B. 2010, Small Atwood number Rayleigh-Taylor experiments, Phil. Trans. R. Soc. A, 368, 1663-1679.

9. Youngs, D.L. 1984, Numerical simulation of turbulent mixing by Rayleigh-Taylor instability, Phys. D, 12, 32-44.

10. Cabot, W.H. \& Cook, A.W. 2006, Reynolds number effects on Rayleigh-Taylor instability with possible implications for type Ia supernovae, Nat. Phys., 2, 562-568.

11. Livescu, D., Ristorcelli, J.R., Gore, R.A., Dean, S.H., Cabot, W.H. \& Cook, A.W. 2009, High-Reynolds number Rayleigh-Taylor turbulence, J. Turb., 10 (13), 1-32.

12. Ristorcelli, J.R. \& Clark, T.T. 2004, Rayleigh-Taylor turbulence: self-similar analysis and direct numerical simulations, J. Fluid Mech., 507, 213-253.

13. Joshi, A., Radhakrishna, M.C. \& Rudraiah, N. 2010, Rayleigh-Taylor instability in dielectric fluids, Phys. Fluids, 22 (064102), 1-10.

14. Wachtor, A.J., Mocko, V., Williams, D.J., Goertz, M.P., \& Jebrail, F.F. 2013, Buoyancy driven mixing of miscible fluids by volumetric energy deposition of microwaves, $J$. Microwave Power \& Electromag. Energy, 47 (3), 210-223.

15. Gabriel, C., Gabriel, S., Grant, E.H., Halstead, B.S.J. \& Mingos, D.M.P. 1998, Dielectric parameters relevant to microwave dielectric heating, Chem. Soc. Rev., 27, 213-223. 
16. Kennedy, A., Reznik, A., Tadesse, S. \& Nunes, J. 2009, Time dependence of component temperatures in microwave heated immiscible liquid mixture, J. Microwave Power \& Electromag. Energy, 43 (2), 52-62.

17. Kennedy, A., Tadesse, S., Nunes, J. \& Reznik, A. 2011, Influence of microwave heating on liquid-liquid phase inversion and temperature rates for immiscible mixtures, J. Microwave Power \& Electromag. Energy, 45 (1), 5-14.

18. Clark, D.E., Sutton, W.H. \& Lewis, D.A. 1997, Microwave processing of materials, Microwaves: Theory and Application in Material Processing IV, Eds. D.E. Clark, W.H. Sutton \& D.A Lewis, The American Ceramic Society, Westerville, OH.

19. Vollmer, M. 2004, Physics of the microwave oven, Phys. Ed., 39 (1), 74-81.

20. Venkatesh, M.S. \& Raghavan, G.S.V. 2004, An overview of microwave processing and dielectric properties of angri-food materials, Biosys. Eng., 88 (1), 1-18.

21. Jindarat, W., Rattanadecho, P. \& Vongparadubchai, S. 2011, Analysis of energy consumption in microwave and convective drying process of multi-layered porous material inside a rectangular wave guide, Exp. Therm. Fluid Sci., 35, 728-737.

22. Sadek, P.C. 2002, The HPLC Solvent Guide, $2^{\text {nd }}$ Edition, John Wiley and Sons, Inc., New York, NY.

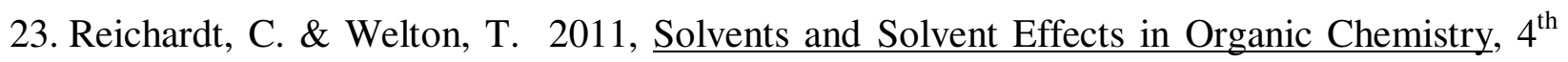
Edition, Wiley-VCH Verlag GmbH \& Co. KGaA, Weinheim, Germany.

24. Abraham, M. 1937, The classical theory of electricity and magnetism, Blackie \& Son Limited, London, UK.

25. Bykov, Y.V., Rybakov, K.I. \& Semenov, V.E. 2001, High-temperature microwave processing of materials, J. Phys. D: Appl. Phys., 34, R55-R75.

26. Kumbharkhane, A.C., Helambe, S.N., Lokhande, M.P., Doraiswamy, S. \& Mehrotra, S.C. 1996, Structural study of aqueous solutions of tetrahydrofuran and acetone mixtures using dielectric relaxation technique, Pramana J. Phys., 46 (2), 91-98. 
27. Chaudhari, A., Khirade, P., Singh, R., Helambe, S.N., Narain, N.K. \& Mehrotra, S.C. 1999, Temperature dependent dielectric relaxation study of tetrahydrofuran in methanol and ethanol at microwave frequency using time domain technique, J. Mol. Liq., 82, 245-253.

28. Zhang, Q., Jackson, T.H. \& Ungan, A. 2000, Numerical modeling of microwave induced natural convection, Int. J. Heat Mass Trans., 43 (12), 2141-2154.

29. Versteeg, H.K. \& Malalasekera, W. 1995, An introduction to computational fluid dynamics, the finite volume method, John Wiley \& Sons Inc., New York, NY. 
Table 1. Fluid properties at standard temperature and pressure. $T_{B P}-$ boiling point; $n$-refractive index; $\mathcal{E}^{\prime}-$ dielectric constant; $\varepsilon^{\prime \prime}-$ dielectric loss; $\tan (\delta)$-loss tangent; $\rho$-density; $C_{p}$-specific heat.

\begin{tabular}{|c|c|c|c|c|c|c|c|c|}
\hline Solvent & $T_{B P}$ & $n$ & $\varepsilon^{\prime}$ & $\varepsilon^{\prime \prime}$ & $\tan (\delta)$ & $\rho$ & $C_{p}$ & $L_{P D}$ \\
\hline & ${ }^{\circ} \mathrm{C}$ & & & & & $\mathrm{g} / \mathrm{cm}^{3}$ & $\mathrm{~J} / \mathrm{g} \cdot \mathrm{K}$ & $\mathrm{cm}$ \\
\hline THF & 66.0 & 1.407 & 7.4 & 0.348 & 0.047 & 0.887 & 1.7153 & 15.3 \\
\hline Toluene & 110.6 & 1.497 & 2.4 & 0.096 & 0.04 & 0.866 & 1.6869 & 31.0 \\
\hline Water & 100 & 1.333 & 80.1 & 9.86 & 0.123 & 0.999 & 4.1813 & 1.7 \\
\hline
\end{tabular}



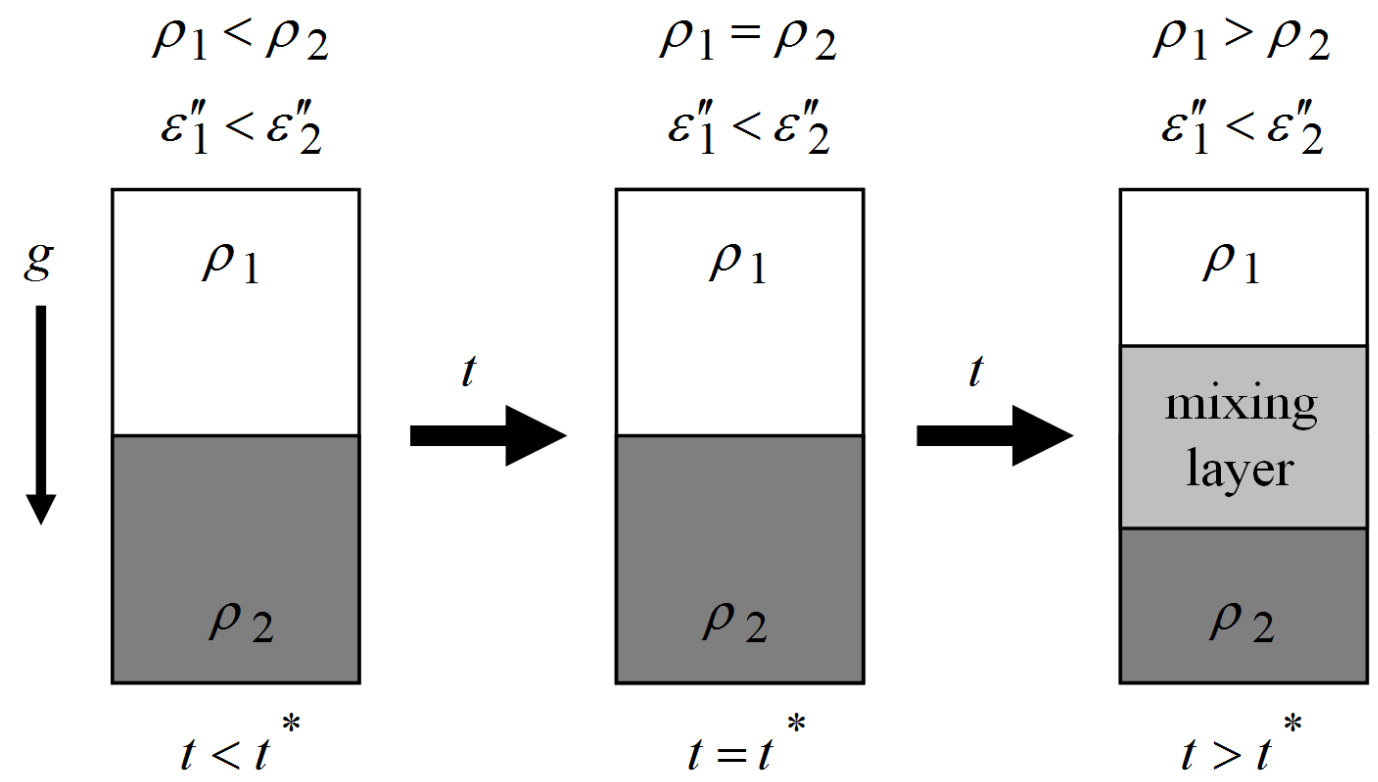

Figure 1: Schematic showing the temporal evolution of the two-fluid system due to subjecting it to volumetric energy deposition. Adapted with permission from [14].

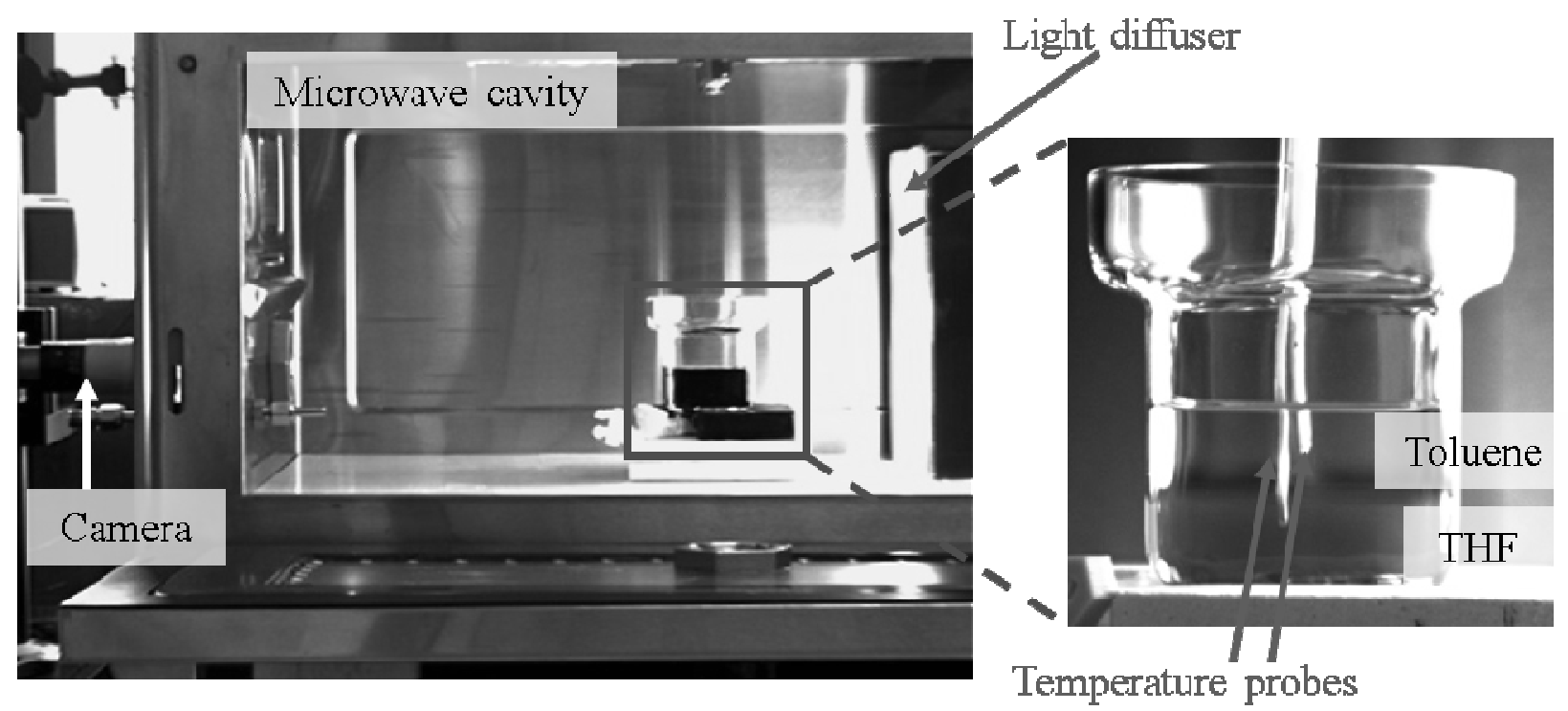

Figure 2: View of experimental facility (left) and close up of containment vessel with temperature probes (right). 


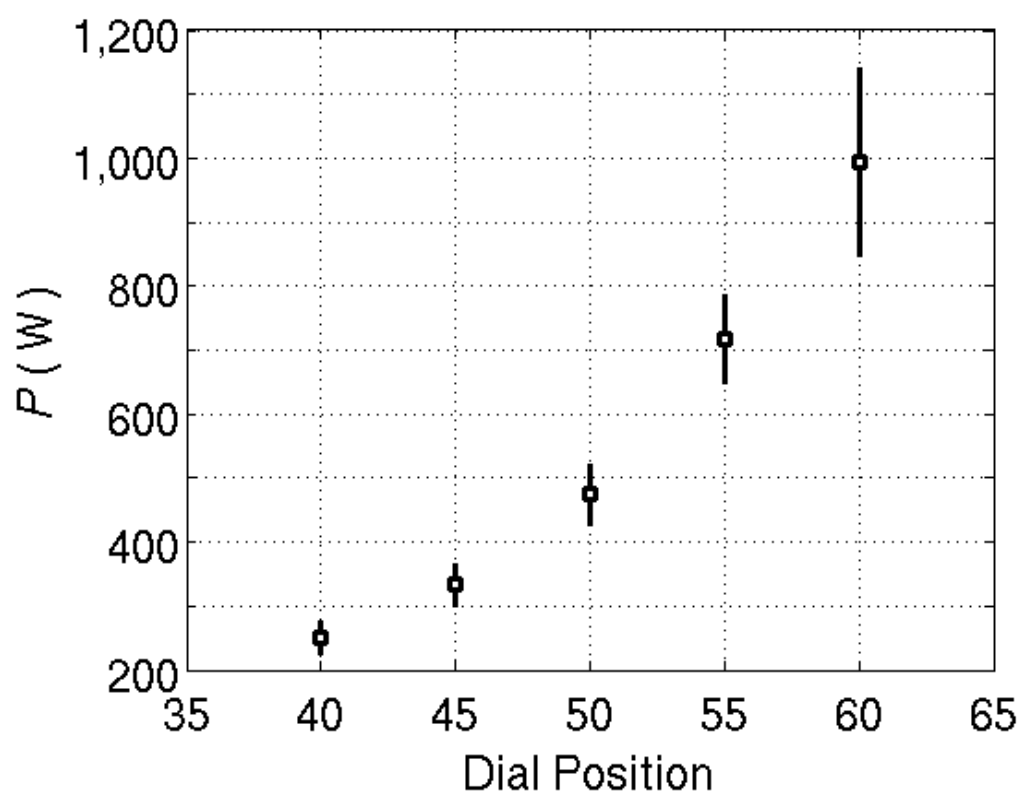

Figure 3: Microwave power calibration using $1 \mathrm{~kg}$ of water. Bars represent one standard deviation from the mean value (Square symbols).

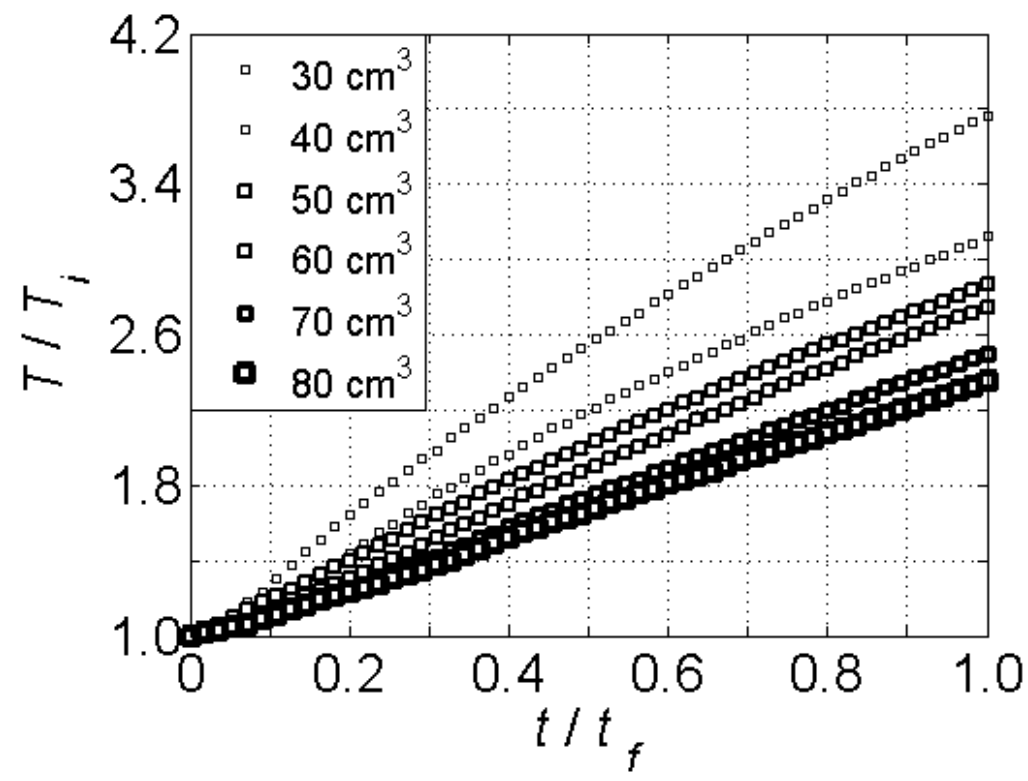

Figure 4: Single fluid temperature measurements showing effect of increasing fluid volume in $10 \mathrm{~cm}^{3}$ increments from 30 to $80 \mathrm{~cm}^{3}$ while heating water at Dial Position 45. Increasing circle size and line thickness represent increasing volume. Non-dimensionalized by (left) initial temperature (right) final temperature rise. 


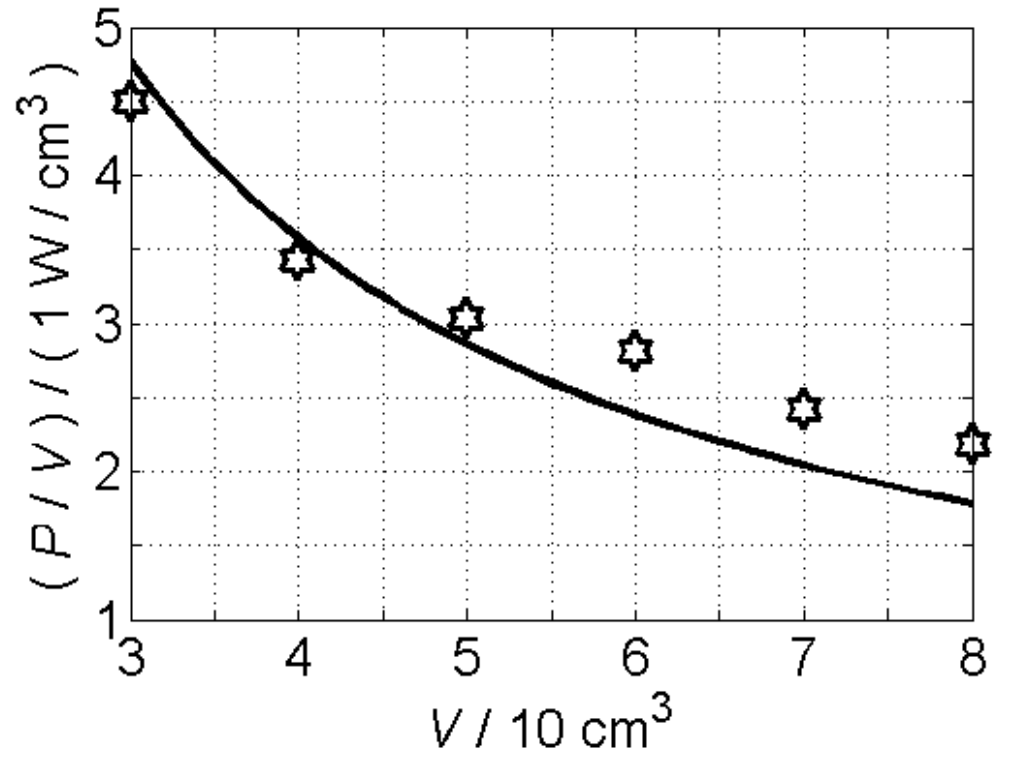

Figure 5: Power absorbed per unit volume vs. volume for water experiments in Figure 4. Power calculated from Equation 1 using $\Delta t=t_{f}=55 \mathrm{~s}$. Line shows behavior based on constant power equal to the mean power measured across all water experiments. 


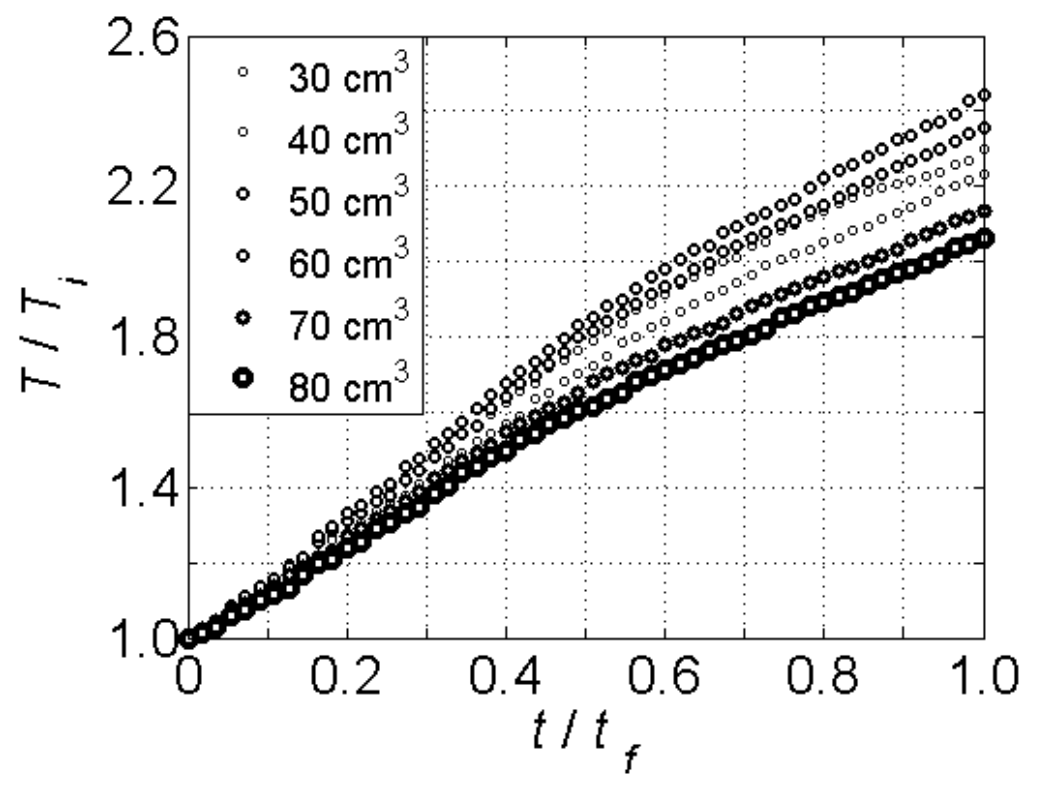

(a)

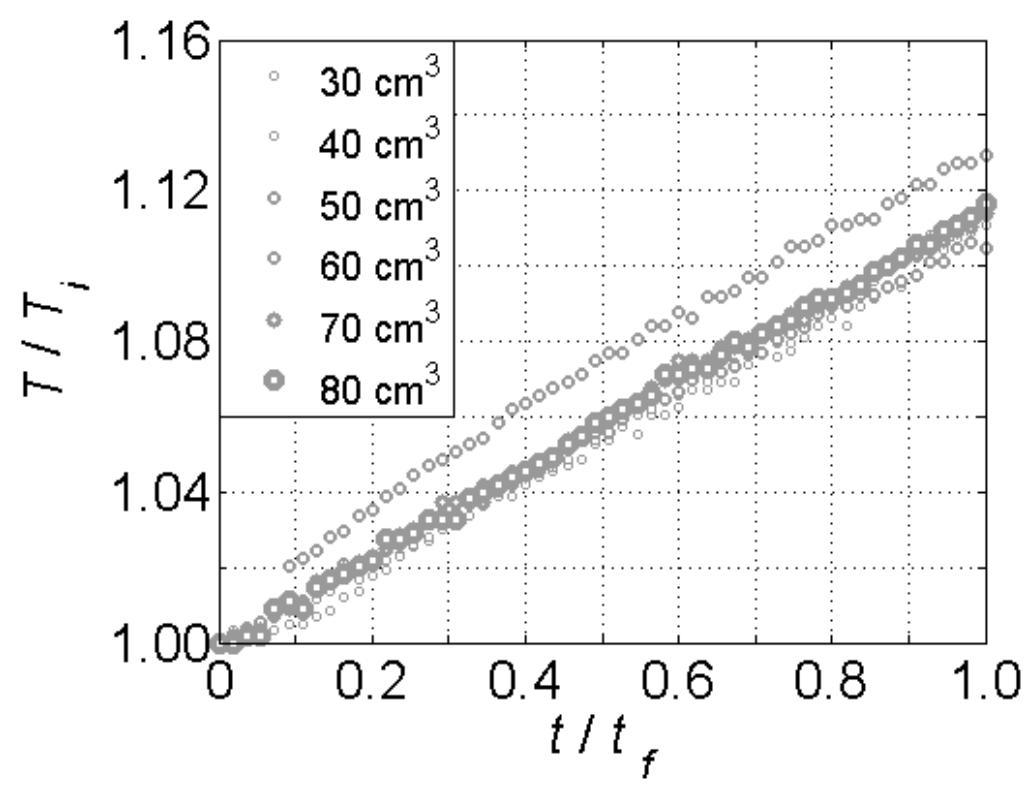

(b)

Figure 6: Single fluid temperature measurements showing effect of increasing fluid volume in $10 \mathrm{~cm}^{3}$ increments from 30 to $80 \mathrm{~cm}^{3}$ while heating (a) THF and (b) toluene at Dial Position 45. Increasing circle size and line thickness represent increasing volume. Axes are non-dimensionalized by initial temperature, $T_{i}$, and total experiment time, $t_{f}$. 


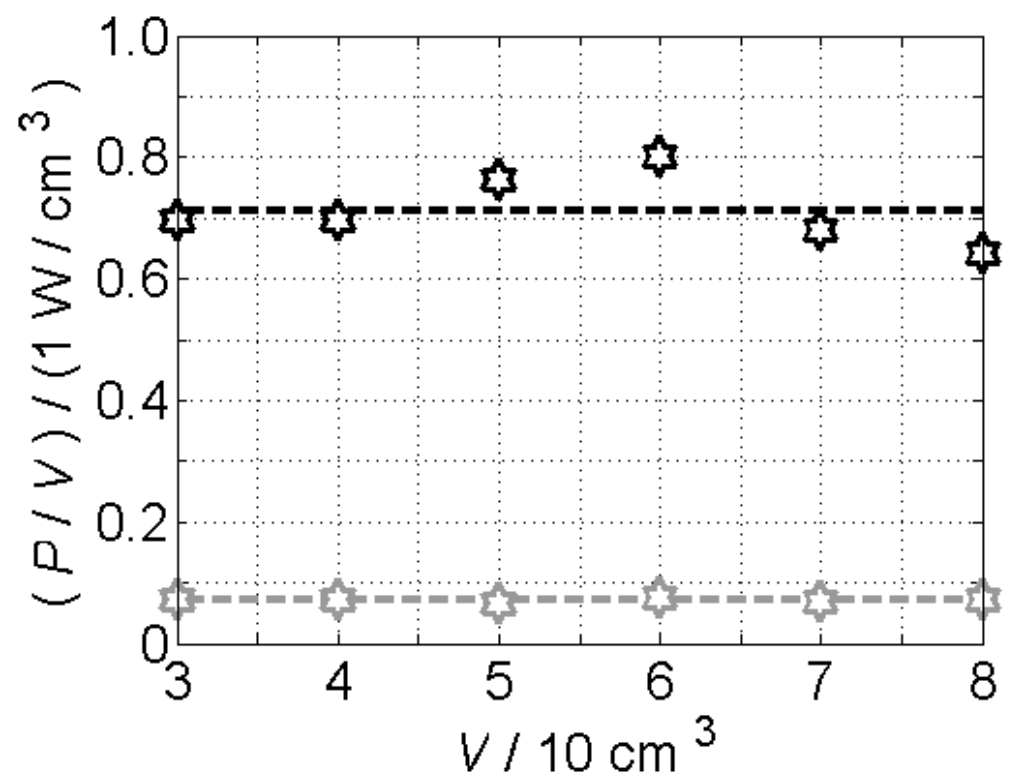

Figure 7: Power absorbed per unit volume vs. volume for measurements in Figure 6. Power calculated from Equation 1 using $\Delta t=t_{f}=55 \mathrm{~s}$. Dashed lines show the average absorbed power density for each of the respective fluids. Black: THF; Gray: toluene.

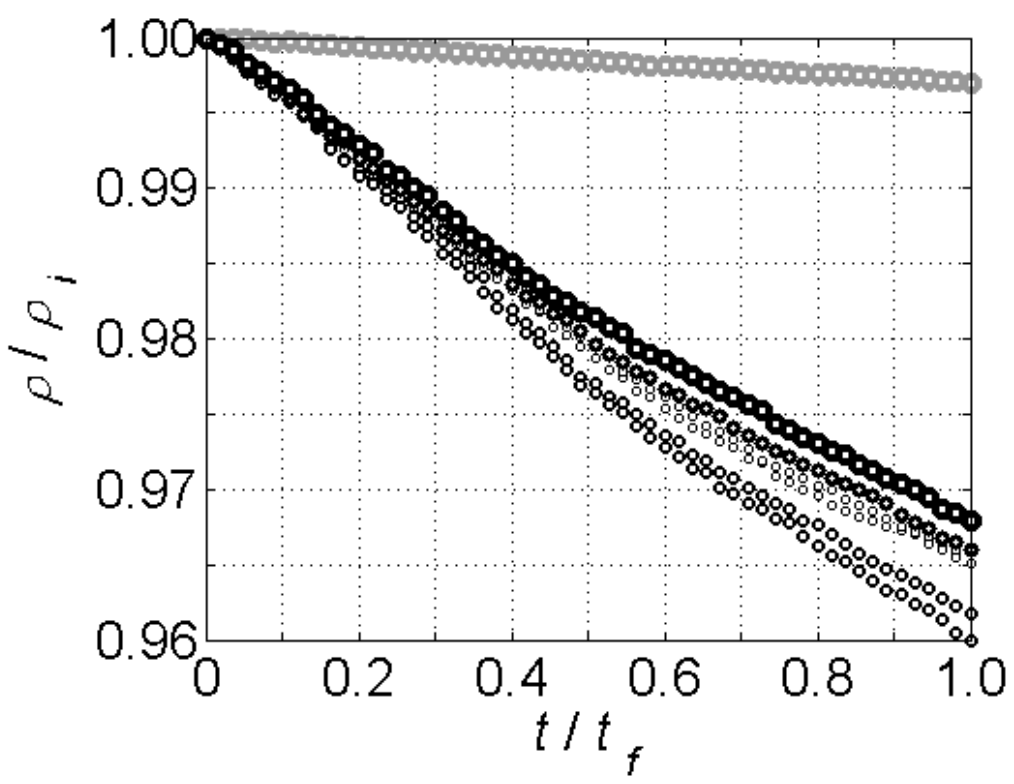

Figure 8: Single fluid density calibration measurements showing effect of increasing fluid volume in $10 \mathrm{~cm}^{3}$ increments from 30 to $80 \mathrm{~cm}^{3}$ while heating at Dial Position 45. Increasing circle size and line thickness represent increasing volume, consistent with Figure 6. Black: THF; Gray: toluene. 


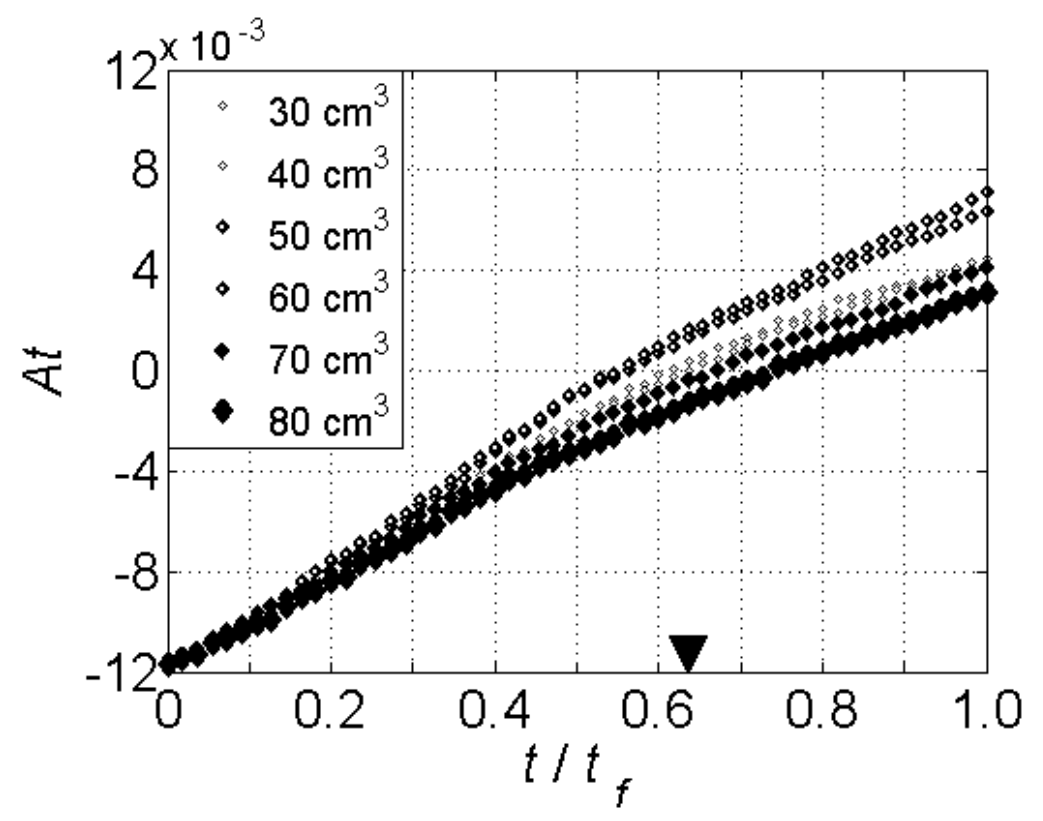

(a)

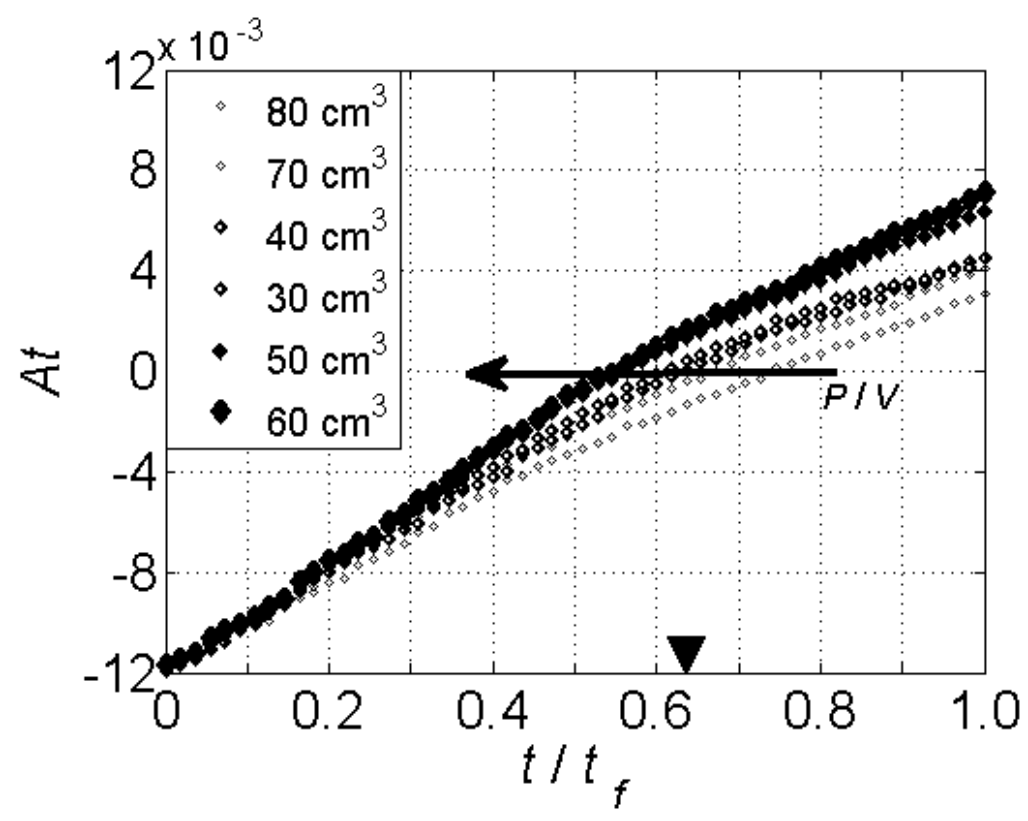

(b)

Figure 9: Temporal evolution of Atwood number, computed from single fluid density calibration measurements, showing the effect of increasing fluid volumes in $10 \mathrm{~cm}^{3}$ increments from 30 to $80 \mathrm{~cm}^{3}$ while heating at Dial Position 45. (a) Increasing diamond size and line thickness represent (a) increasing volume and (b) increasing absorbed power density. Marker shows neutral stability point prediction for $40 \mathrm{~cm}^{3}$ measurements 


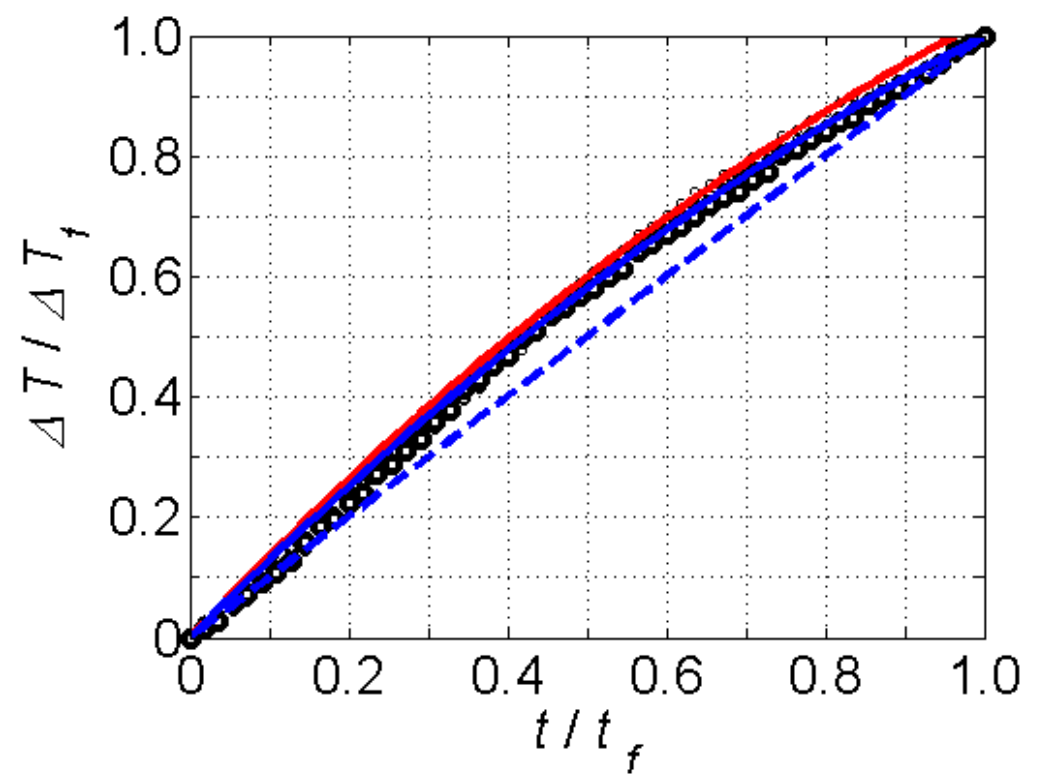

(a)

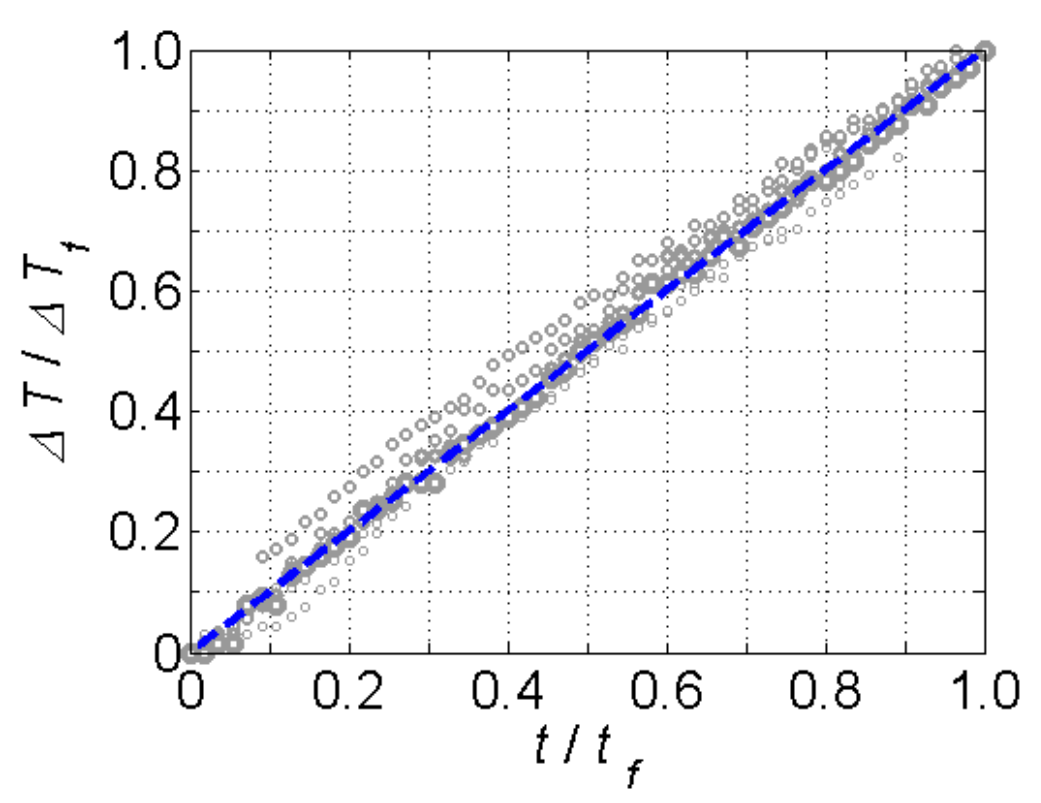

(b)

Figure 10: Temperature rise measured while heating at Dial Position 45 and increasing fluid volume in $10 \mathrm{~cm}^{3}$ increments from 30 to $80 \mathrm{~cm}^{3}$ vs. time from experiments (symbols) compared with model solutions (blue dashedlines: constant power model, blue solid-line: linear power model) and analytical solution (red solid-line: exponential solution from Equation 15). Increasing circle size and line thickness represent increasing volume. (a) THF (b) toluene. 


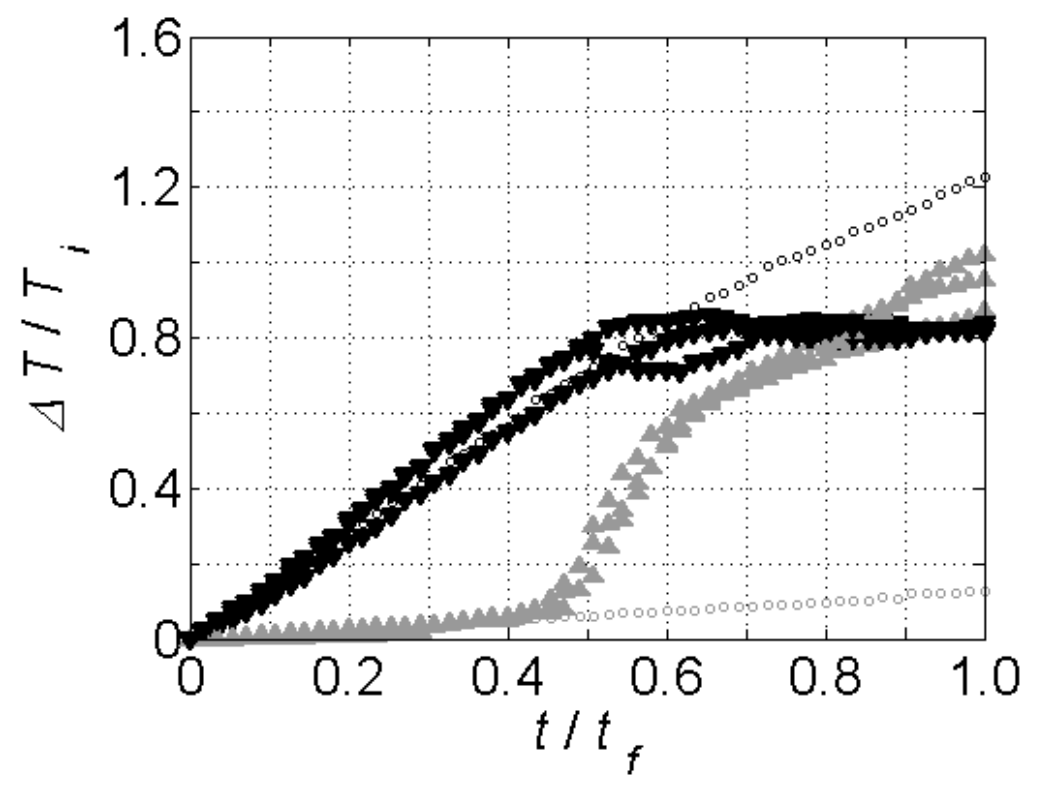

(a)

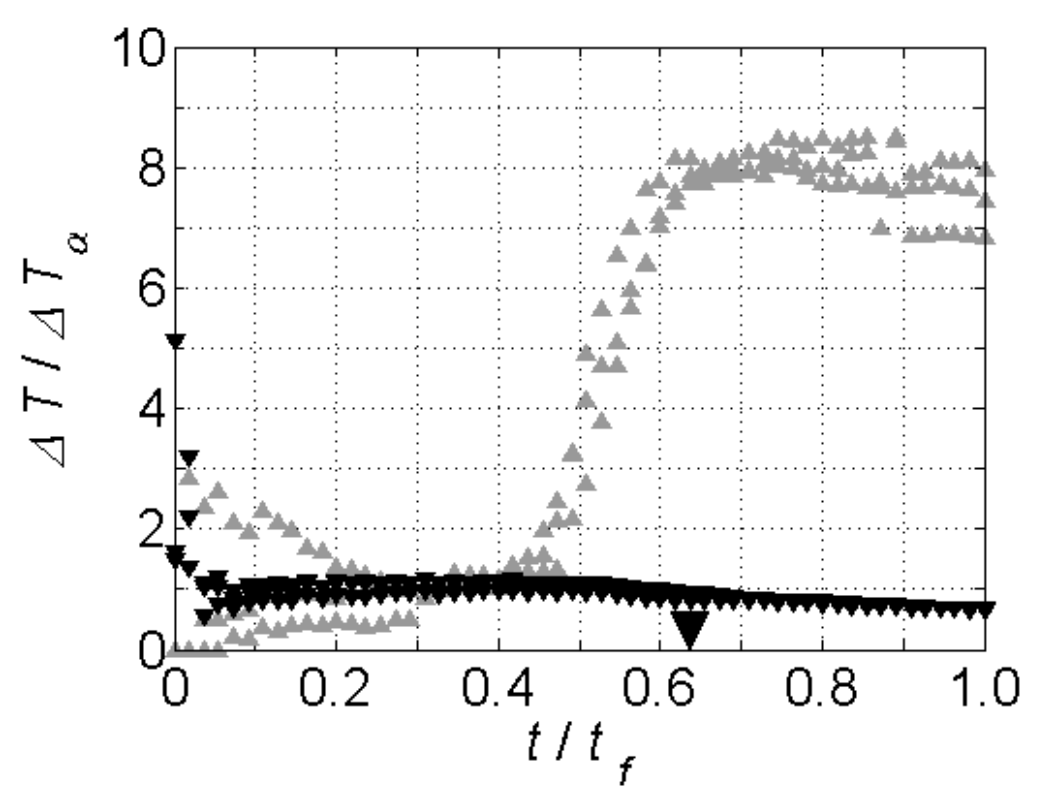

(b)

Figure 11: Temperature rise measurements from two-fluid mixing experiments of $40 \mathrm{~cm}^{3}$ toluene $/ 40 \mathrm{~cm}^{3} \mathrm{THF}$ heated at Dial Position 45, non-dimensionalized by $(a)$ initial temperature, $T_{i}$, and $(b)$ the temperature rise of the respective $40 \mathrm{~cm}^{3}$ pure fluid initially at the probe location. Black downward triangle: Bottom probe (initially in THF), Gray upward triangle: Top probe (initially toluene). Data from three experiments shown to demonstrate repeatability. Temporal axis non-dimensionalized by total time of fluid experiment. 

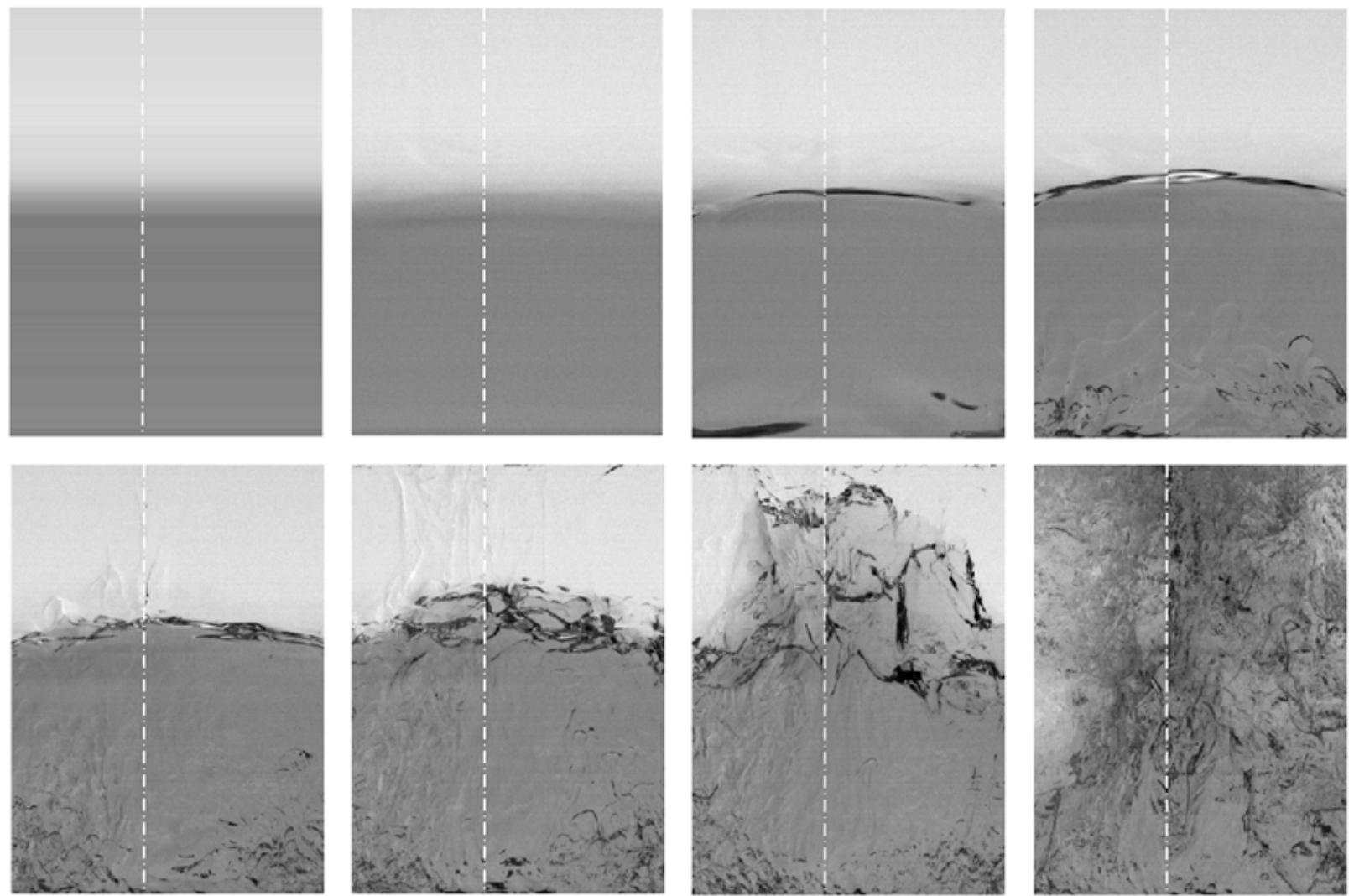

Figure 12: Images showing the temporal evolution of the two-fluid mixing process at Dial Position $45, \Delta t=0.07 t_{f}$ between images. Each image has been corrected for uniform backlighting.

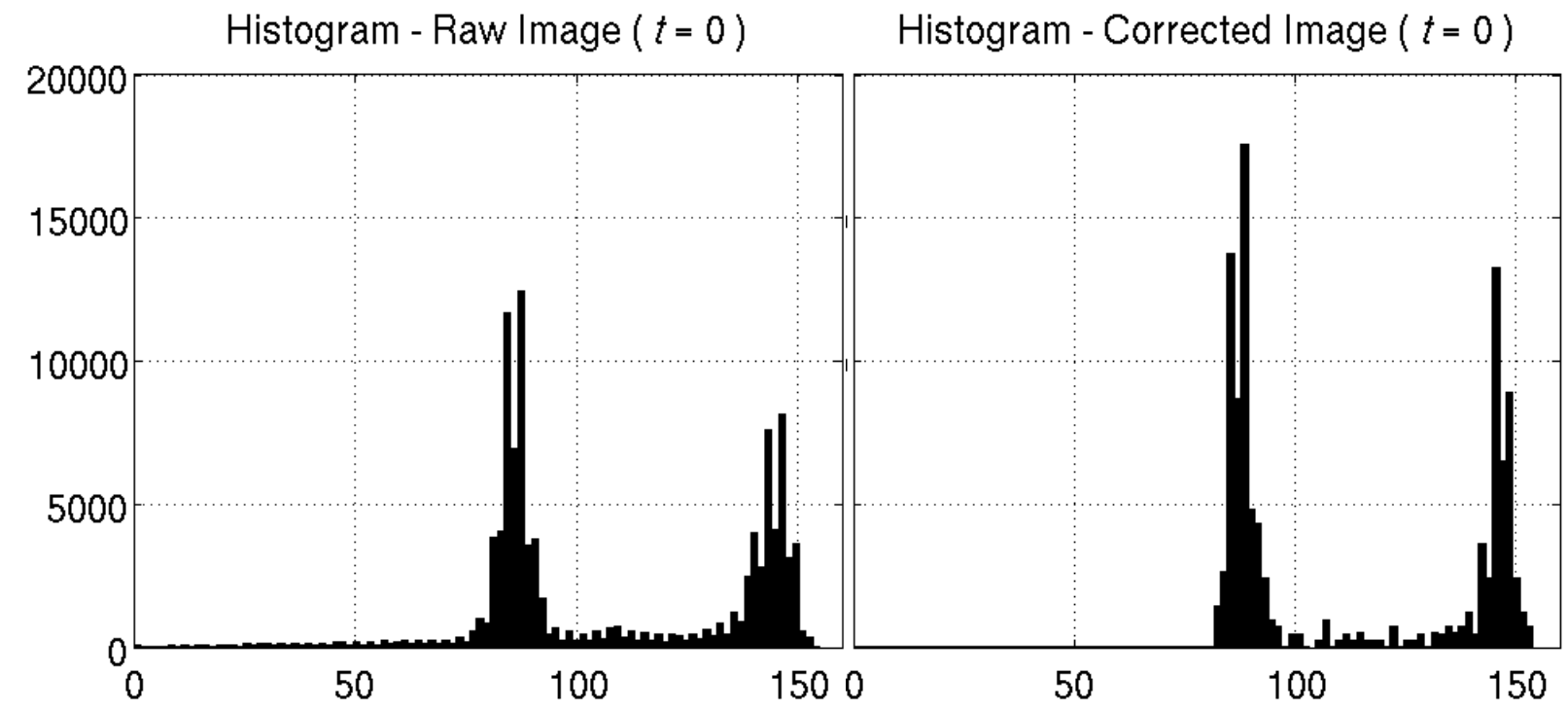

Figure 13: Histogram of raw image and image corrected for uniform backlighting (with probes omitted) at $t=0.0$. 


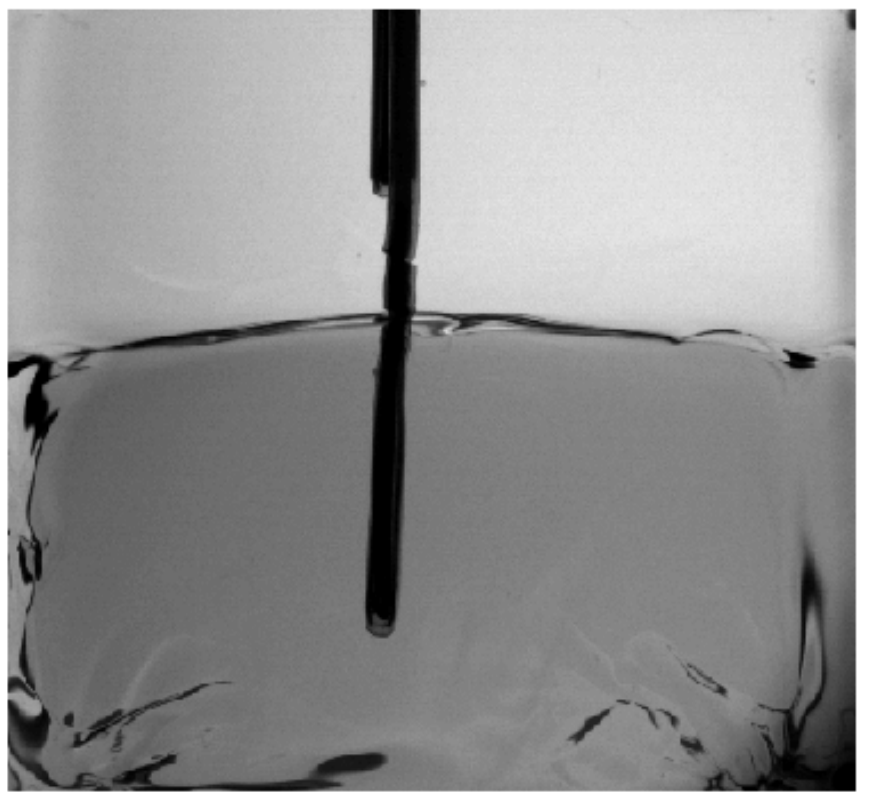

(a)

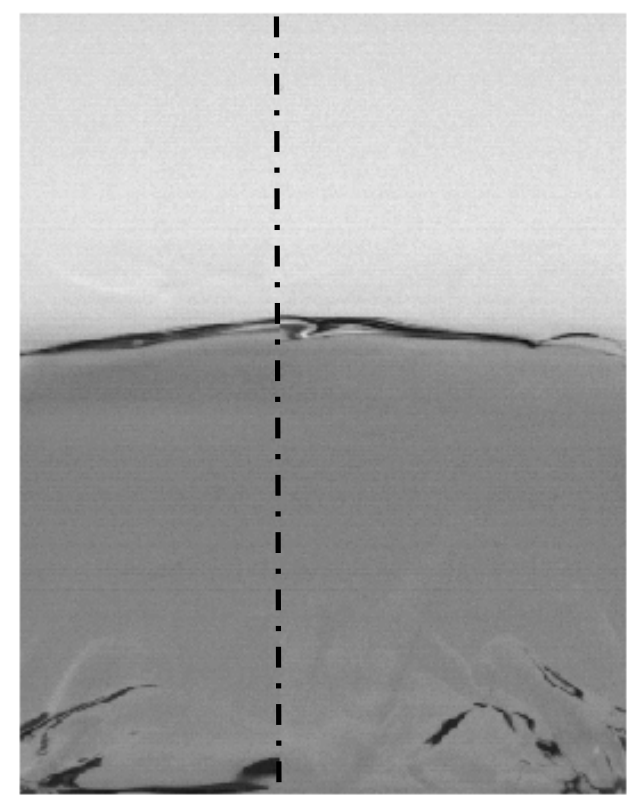

(c)

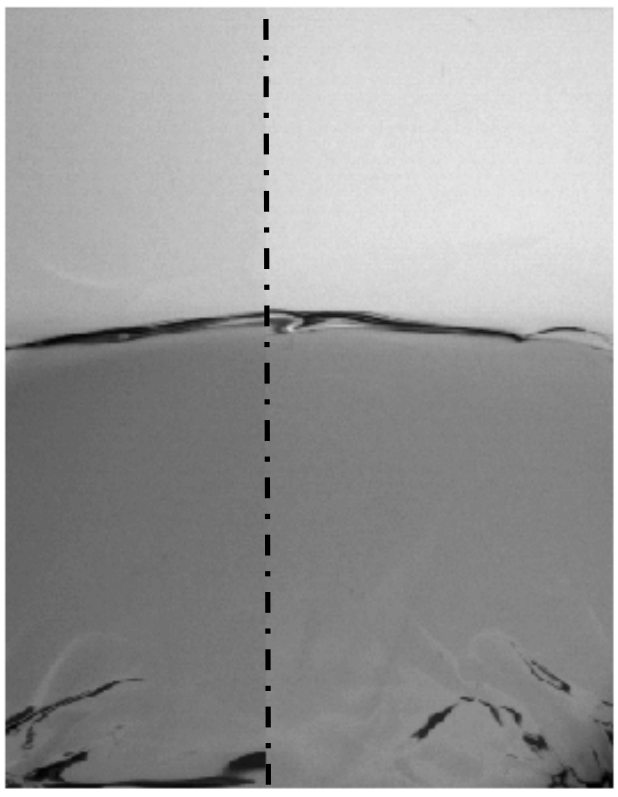

(b)

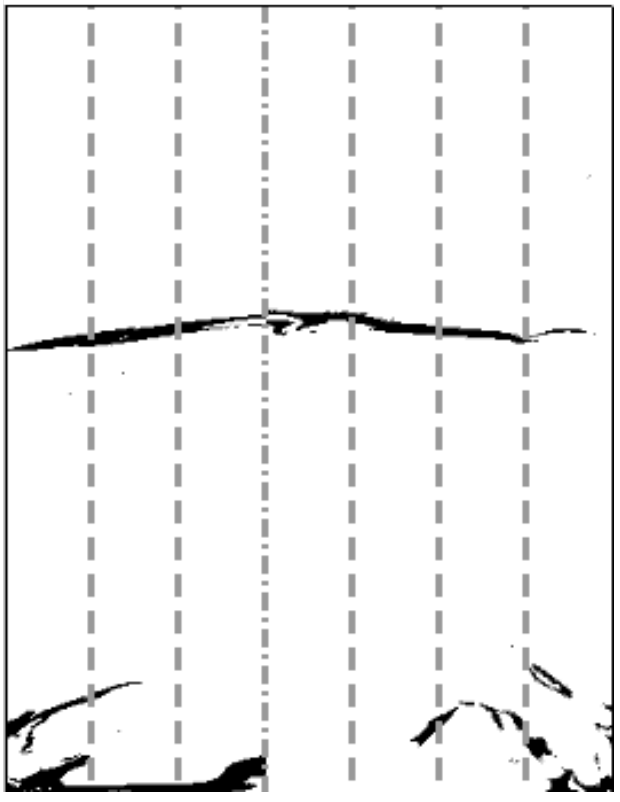

(d)

Figure 14: (a) Typical raw image collected from experimental setup. (b) Same image as (a) with probes omitted. (c) Same image as (b) with image corrected for uniform backlighting. (d) Thresholded image derived from (c). Vertical dash-dotted line represents the location where the probes were omitted. Vertical dashed lines represent the subsections the image was divided into for analysis. 


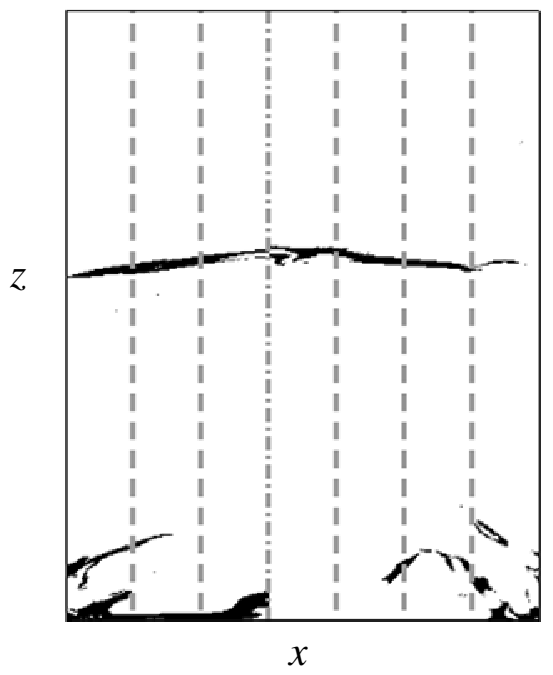

Normalized Average Profile for Each Subsection

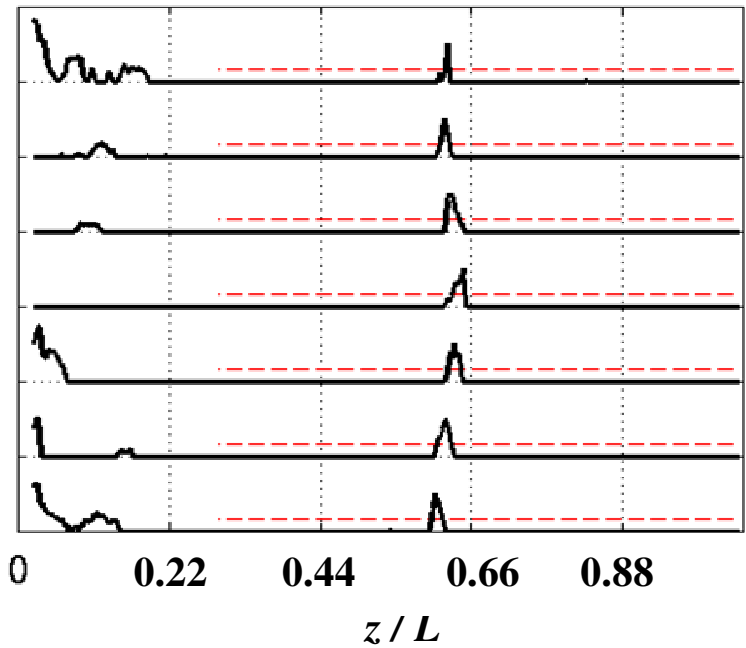

(a)
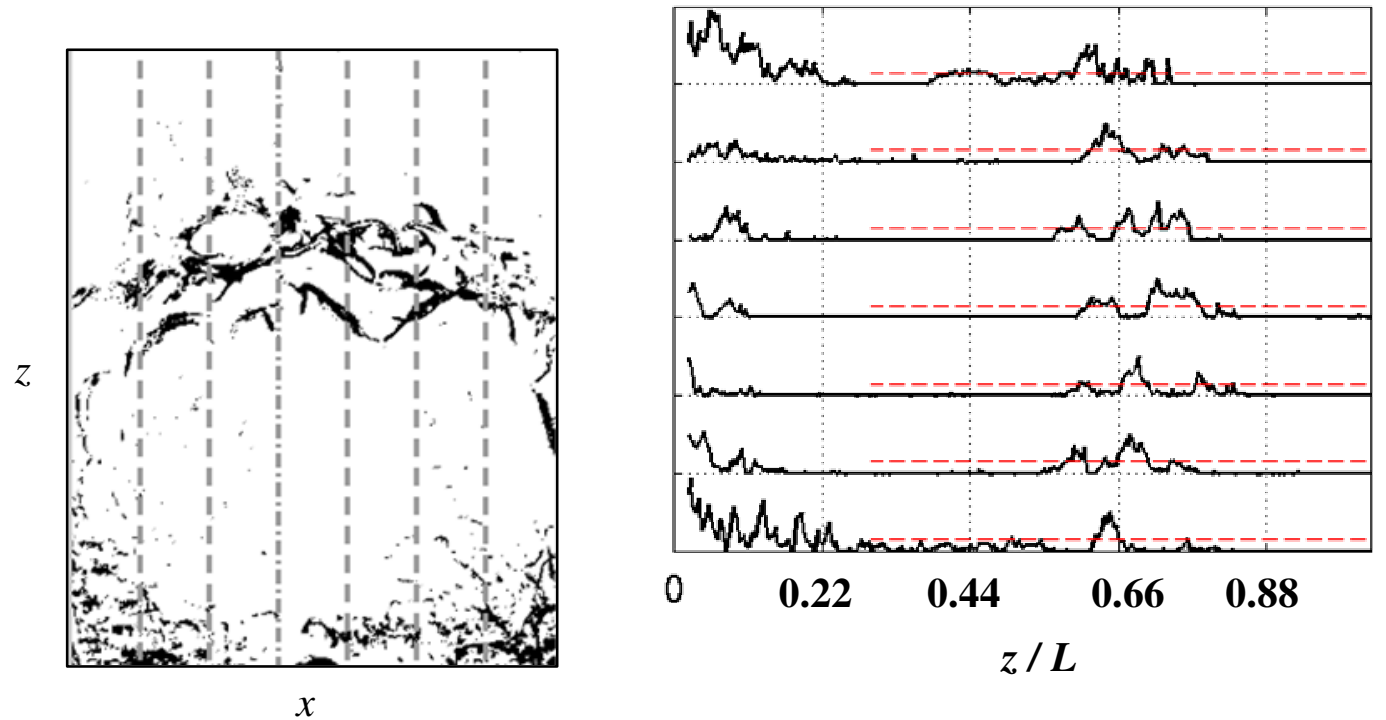

(b)

Figure 15: Examples of thresholded images from mixing experiment (left) and corresponding curves from which mixing layer width is computed (right). The curves on the right represent the integration over $x$ for each subsection which has been normalized by the peak value (found outside the bottom portion of the vessel were index of refraction issues are present in the heating of THF). The mixing layer estimation is then determined by finding the distance between the lowest and highest physical location for which the curve surpasses $30 \%$ of the peak value (again omitting the bottom portion where the mixing layer is known not to be). (a) $0.24 t_{f}$ (b) $0.52 t_{f}$. 


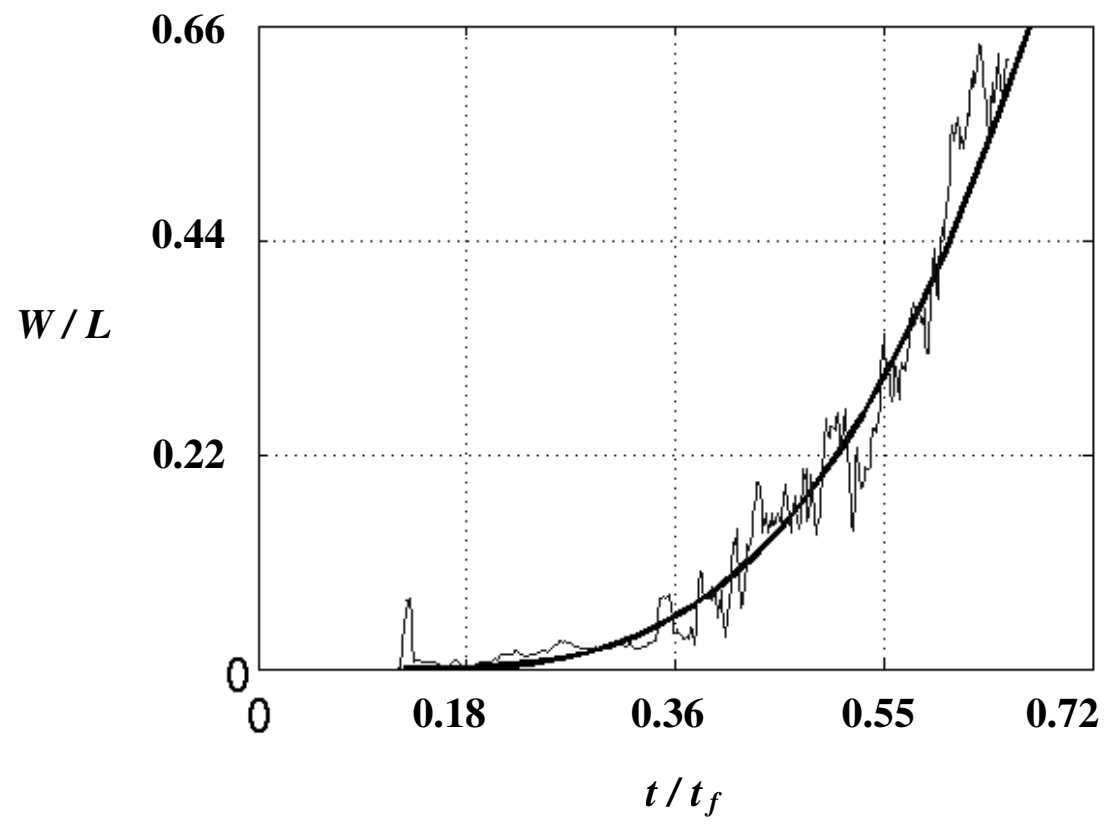

Figure 16: Temporal evolution of the mixing layer width. Thin line: estimation based on image processing; thick line: theoretical $\sim t^{3}$ fit. 